\title{
GENERALIZATIONS OF THE FIRST AXIOM OF COUNTABILITY
}

\author{
FRANK SIWIEC
}

Abstract. This paper is a comprehensive survey of the concepts which generalize first countability, of the relations among the concepts, and of the major examples found in the literature on the topic.

0. Introduction. There are several reasons which one may give to indicate the value of generalizing the first axiom of countability. Among these are the following:

(1) To weaken assumptions in important theorems. For example, a closed image of a metric space is metrizable, if the range is assumed to be first countable.

(2) To study important properties. For example, if a real valued function $f$ is continuous upon restriction to each compact subspace of a space $X$, then $f$ is continuous on $X$, if $X$ is a $k$-space.

(3) To study sequences and their properties. For example, in first countable spaces every accumulation point of a subset $A$ is the limit of a sequence in $A$.

The emphasis in this article is on giving a comprehensive list of concepts which have been introduced to generalize first countability, from different points of view, along with examples and references. The reader may often judge the value of a concept by considering the number of references pertaining to it, as listed in our references in Section 1.

The structure of this survey is as follows: In Section 1, we present a list of definitions of concepts which generalize first countability. For standard terminology we follow Kelley [199], Nagata [290], and Thron [379], and the reader may note that there are some remarks concerning notation and terminology at the beginning of Section 1. The reader is advised to skim Section 1 and refer to it as needed as he would a dictionary. The history of the subject is briefly surveyed in Section 2, along with some motivation for the concepts and some

AMS Subject Classification (1970): Primary 54D50, 54D55.

Much of the work on this paper was done while the writer was at St. John's University, Jamaica, New York.

Received by the Editors May 20, 1971 and, in revised form, January 31, 1974.

Copyright $\odot 1975$ Rocky Mountain Mathematics Consortium 
mention of unsolved problems. Section 3 gives implications and equivalences among the axioms discussed. In Section 4 , we give a list of examples, all of which are both Hausdorff and not first countable. This list may serve the purpose of establishing which of the classes of spaces are distinct. The paper concludes with an exhaustive bibliography on the subject.

All spaces are understood to be Hausdorff spaces, unless otherwise stated, with the following exception: In Section 1 we define the concepts without assuming the Hausdorff axiom. It should be noted that in some of the original articles some separation axiom is taken as part of the definition of the concept. Thus, definitions in the original papers may at times differ, though they are equivalent upon assuming the Hausdorff property.

Finally, we would like to add that as in any other rapidly developing field, terminology tends to vary, and we hope this survey will serve as an incentive for standardization.

1. The Concepts. In this section we give what we believe to be a comprehensive list of the concepts found in the literature which generalize first countability. First, however, it is desirable for us to discuss some terminology used in this paper.

The following conventions will be used unless stated otherwise: The word mapping will mean a continuous surjection; the letter $X$ will stand for a topological space (see the remarks concerning the assumption of the Hausdorff axiom in the Introduction); $N$ will stand for the set of positive integers, while letters such as $i, j, k, m, n$ will represent elements of $N$, and $c$ will represent the cardinality of the continuum. The symbol "iff" is an abbreviation for "if and only if"; "+" will mean that two (or more) properties are held simultaneously; " $=$ " will mean that two classes of spaces are equivalent. The boundary, closure, and interior of a set, will be denoted by "Bdy", "Cl", and "Int", respectively.

The words accumulation point and cluster point will be used in the following senses: A point $x$ is an accumulation point (or synonymously limit point) of a set $A$ if every neighborhood $U$ of $x$ meets $A$ in a point distinct from $x$. A point $x$ is a cluster point of a sequence $\left\{x_{n}\right\}$ if for every neighborhood $U$ of $x$ and every positive integer $m$, there exists an integer $n>m$ such that $x_{n} \in U$. By extension, the term cluster point will also be used in cases involving nets and filters in the usual sense. (This terminology is consistent with that used in most topology textbooks.) A sequence (or net) is eventually in a set $U$ if $U$ contains all but finitely many elements of the sequence (or net). Following Michael [267], we say that a filter base 7 converges to a set A (where 
$A$ is permitted to be a one point set) if every neighborhood of $A$ contains some $F \in \mathcal{Y}$. A sequence $\left\{A_{n}\right\}$ of sets is decreasing if $A_{n} \supset A_{n+1}$ for all $n$ in $N$. A decreasing sequence $\left\{A_{n}\right\}$ of non-empty sets is a $k$-sequence (respectively, q-sequence) if it converges to some compact (respectively, countably compact) set which is contained in $A_{n}$ for all $n$. In the following, a slash, $l$, separates the major references from the minor references.

A space $X$ is an absolute Fréchet-Uryson space iff whenever $x \in X$, $M \subset \beta X$ and $x \in \mathrm{Cl}_{\beta X} M$, there exists a sequence of points in $M$ converging to $x$. A bi-sequential space is absolute Fréchet-Uryson, and such a space is countably bi-sequential. Arhangel'skii [31].

For a space $X$ and for any class $C$ of subsets of $X$, an accumulation point $x$ of a set $M$ is accessible by C-sets provided $x$ is an accumulation point of a $C$-set lying in $M \cup\{x\}$. See: approximately accessible by $C$-sets, property $H$, property $K$, and also accessibility space. Whyburn $[399] /[346]$.

A space $X$ is an accessibility space iff every accumulation point $x$ of a set $M$ in $X$ is approximately accessible by closed sets, that is, there is a closed set $C$ such that $x$ is an accumulation point of $C$ but not of $C-M$. For a $T_{1}$-space $X, X$ is an accessibility space iff it is a pseudoopen space; property $H$ implies accessibility space; and for a regular space $X, X$ is an accessibility space iff it has property $H$. Another related concept is Aull's $Z$ space. Whyburn [399]/[44, 238, 346, 352, $354,356]$.

A space $X$ is said to be accumulation complete iff each sequence that has a point $x$ as a cluster point, has a subsequence that converges to $x$. A space being accumulation complete is equivalent to every countable subspace being Fréchet. This concept has also been called subsequential in [373]. An accumulation complete space is an $\mathrm{H}_{2}$ space. [ $175,267,323,354,373]$.

A space satisfying property $\alpha$ is the former terminology of Whyburn in [398] for a $k^{\prime}$-space.

For a space $X$ and for any class $C$ of subsets of $X$, an accumulation point $x$ of a set $M$ is approximately accessible by $C$-sets provided there exists a $C$-set $C$ having $x$ as an accumulation point and such that all points of $C$ in some neighborhood of $x$ lie in $M \cup\{x\}$. See also: accessible by $C$-sets, accessibility space. Whyburn [399] .

A space $X$ is a $b_{R}$-space iff every real-valued function whose restriction to each relatively pseudocompact subset $K$ is continuous on $K$, is continuous on $X$, where a subset $K$ is said to be relatively pseudocompact in $X$ iff each continuous real-valued function on $X$ is bounded on $K$. Noble [302] . 
The definition of a $b_{R}{ }^{*}$-space is analogous to that of a $b_{R}$-space with the term "relatively pseudocompact subset" replaced by "subset whose product with each pseudocompact space is relatively pseudocompact in the product". The class of spaces which are $b_{R}{ }^{*}$ is between the classes of spaces which are $k_{R}$ and $b_{R}$. Noble [302].

A space $X$ is a $b i-k$ space iff whenever a filter base $\exists$ has a cluster point $x$ in $X$, there exists a $k$-sequence $\left\{A_{n}\right\}$ in $X$ such that $F$ intersects $A_{n}$ for all $n$ and all $F \in \mathcal{F}$. This terminology was formerly used for the concept of a bi-quasi- $k$ space by Nagata in [292]. Michael $[267] /[31,170,312,336,337,351,353]$.

The definition of a bi-quasi-k space is the same as the definition of a bi- $k$ space with the term " $k$-sequence" replaced by " $q$-sequence". This concept was formerly called a bi-k space by Nagata in [292]. Michael [267], Nagata [292]/[312,336,337].

A space $X$ is a bi-sequential space iff whenever a filter base $\mathcal{Y}$ has a cluster point $x$ in $X$, then there is a decreasing sequence $\left\{A_{n}\right\}$ of sets in $X$ converging to $x$ and such that $F$ intersects $A_{n}$ for all $n$ and for all $F \in$ 7. Michael [267], Arhangel'skii [31], Malyhin [233], Olson $[312] /[228,235,236,336,337,351,352,353]$.

The terminology c-space has been used for at least three concepts. (1) A space $X$ is a $c$-space iff for each non-isolated point $x$ of $X$ and each sequence $\left\{U_{n}\right\}$ of neighborhoods of $x$, there exists a set $\left\{x_{n} \mid n \in N\right\}$ which is not closed and is such that $x_{n} \in U_{n}$ for every $n$. Every $k$-space is a $c$-space in this sense. Chaber [87]/[355]. (2) A space is a $c$-space iff the closed sets are exactly those for which the intersection with every compact closed set is compact. Assuming the Hausdorff property, this is equivalent to the concept of a $k$-space. de Groot $[140] /[17,18,45,327,339]$. (3) This terminology is also in use $[331,122,335,336]$ for spaces determined by countable subsets. See: countable tightness.

A space satisfies condition $\left(C_{0}\right)$ iff each sequence whose closure is countably compact has a subsequence whose closure is compact. This is implied by condition $\left(k_{0}\right)$ of Chiba. Tanaka [375] .

A space being of type $C$ is Noble's [304] terminology for a space of point countable type.

A space $X$ is of type $C^{*}$ iff there exists a cover $C_{0}$ of subsets of $X$ which are compact + first countable + have countable character in $X$ and $C_{0}$ has the following property: for each $x$ in $X$, each $C$ in $C_{0}$ for which $x$ is in $C$, and each neighborhood $U$ of $x$, there exists a $C^{\prime}$ in $C_{0}$ with $x \in C^{\prime} \subset U \cap C$. For Hausdorff spaces, this is equivalent to first countability (by (d) of (20) of Section 3). Noble [304].

The character of a set $A$ in a space $X$, denoted by $\chi(A, X)$, is the least cardinality of a collection $\mathcal{B}$ of open neighborhoods of $A$ having the 
property that every neighborhood of $A$ contains some member of $\mathcal{B}$. The character of a space $X$, denoted by $X(X)$, is the supremum of the characters of all points of $X$. Other terminologies are: local character, local weight, point character, pointwise weight, weight; compare: pseudo character. This concept was discussed in the 1929 memoir of Alexandroff and Urysohn [2]. A few recent references are: $[26,177,187,195,247,250,251,282,283,315,316,318,322]$.

The terminology closure-countable is used by Wilansky in [406, p. 292] for the concept of countable tightness.

The terminology closure-sequential is that of Wilansky in [406, p. 292] for a Fréchet space.

The terminology compactly-generated space is in common use among algebraic topologists (e.g., Spanier in [360]) and others (e.g., Herrlich [170] ) for a $k$-space.

A space $X$ has countable tightness iff whenever a point $x$ is in the closure of a set $A$ in $X$, there is a countable subset $C$ of $A$ such that $x$ is in the closure of $C$. Equivalent conditions are: the closure of each set in $X$ is the union of the closures of its countable subsets; and if $A$ is a set in $X$ and $\mathrm{Cl} C \subset A$ for every countable subset $C$ of $A$, then $A$ is closed in $X$. The latter condition is the version in which this concept was first introduced by Moore and Mrowka in [273] under the name: a space determined by countable subsets. This terminology is in common use. Other names for this concept are: $c$-space, closurecountable space, countably generated space, countably accessible space (and formerly, space with an $\boldsymbol{\aleph}_{0}$-topology [219]). The concept of tightness, for arbitrary cardinality, was introduced by Arhangel'skii and Ponomarev in [35] and has been discussed frequently in Arhangel'skii's recent work. Compactification: $[31,233]$, examples: [126, 188], general theory: [54, 187, 286], lattice of topologies: [218, 219, $221]$, relation to mappings: [267, $268,335,353]$, relation to products: $[31,212,233]$, relations to other cardinal functions: $[28,29,30,31,32$, $33,35,250,342,343,344]$, other: [122, 125, 330, 331, 334, 335, 336, 406] .

Countably accessible space is the terminology of $[218,221]$ for a space of countable tightness.

A space $X$ is a countably bi-k space iff whenever $\left\{F_{n}\right\}$ is a decreasing sequence of sets in $X$, having $x$ as a common accumulation point, then there is a $k$-sequence $\left\{A_{n}\right\}$ such that $x \in \operatorname{Cl}\left(A_{n} \cap F_{n}\right)$ for all $n$. Michael $[267] /[31,312,336,351,353]$. A somewhat related property is mentioned in [228, p. 46].

The definition of a countably bi-quasi-k space is the same as the definition of a countably bi- $k$ space with the term " $k$-sequence" replaced by " $q$-sequence". Michael [267]/[278, 312, 336, 355]. 
A space $X$ is a countably bi-sequential space iff whenever $\left\{F_{n}\right\}$ is a decreasing sequence of sets in $X$ having $x$ as a common accumulation point, there exists a decreasing sequence $\left\{A_{n}\right\}$ which converges to $x$ and such that $A_{n}$ intersects $F_{n}$ for all $n$. Equivalently, the conclusion of the preceding sentence may read: there exist points $x_{n} \in F_{n}$ such that $x_{n} \rightarrow x$. The latter version was called strongly Fréchet in [352]. Michael [267], Siwiec [352], Arhangel'skii [31], Malyhin [233], Olson [312]/[336, 351, 353,355] .

The terminology countably generated space is that of Franklin and Kohli [125] for a space of countable tightness.

A space $X$ is determined by countable closed subsets iff every set $A$ in $X$ which contains the closure of every subset $B$ of $A$ such that $\mathrm{Cl} B$ is countable, is closed in X. Moore and Mrowka [273], other: [122, $187,188,286]$.

For the concept of a space determined by countable subsets, see: countable tightness.

A space being determined by sequences is the terminology of Moore and Mrowka [273] for a sequential space.

A space $X$ is a $D N$-space iff for each $x$ in $X$ there is a net in $X-\{x\}$ that converges to $x$ and has the property that its range is a discrete subspace of $X$. Anderson [4] .

The terminology E-space is that of McDougle [181] for a Fréchet space.

For the concept of an $E_{0}$-space, see: space in which each point is a $G_{\delta}$-set.

A space $X$ is an $E_{1}$-space iff every point of $X$ is a countable intersection of closed neighborhoods. Aull [37]/[81, 124, 349, 350, 355].

Hausdorffs terminology for a space satisfying the first axiom of countability is nowadays commonly shortened to a first countable space.

A space $X$ is a first countable space iff it has countable character, that is, every point has a countable open base for its neighborhoods. Other terminology: space satisfying the first axiom of countability, space with a locally countable base, locally separable space, weakly separable space.

A space $X$ is Fréchet iff whenever a point $x$ is an accumulation point of a set $A$, there is a sequence in $A$ which converges to $x$. This concept has also been called: closure-sequential space, $E$-space, Fréchet-Urysohn space, $F U$ space. The latter two terms are standard among Russian topologists. Otherwise the term Fréchet space is standard, though the Russian terminology might be used where confusion is possible with another meaning of Fréchet space. For Haus- 
dorff spaces, the following are equivalent: Fréchet space, hereditarily $k$-space (i.e., every subspace is a $k$-space), space with property $K$. The major references are Franklin's [118 and 120] .

Characterizations: $[13,15,19,23,44,102,118,146,157,175,187$, $225,233,248,250,312,337,351,352,353,370,371]$.

Compact spaces: $[35,41,108,110,111,239]$.

Dimension: [414].

Examples: $[4,9,15,30,34,39,41,49,102,112,119,120,126,128$, $133,166,192,212,233,244,267,304,310,312,313,358]$.

Function spaces: [102, 143, 246, 248].

General theory: [54, 121, 187, 190].

Mappings: $[13,15,19,23,54,117,118,127,146,150,154,157,168$, $205,222,223,232,238,243,244,250,267,304,312,336,337,346,351$, $352,353,355,370,371,413]$.

Metrization: [108, 141, 154, 239, 354] .

Modifications of a Fréchet space: $[34,157,168,205,206,247,249$, 250, 253, 254, 287].

Paracompactness: $[63,64,212]$.

Products: $[31,118,120,127,143,154,155,212,238,267,304,310$, 313].

Relations to other generalizations of first countability: $[18,19,23$, $31,34,39,41,44,102,117,118,141,157,166,168,175,182,187,190$, $192,233,244,246,247,248,249,250,253,346,352,358,399]$.

Survey remarks: $[18,30,267,336,399]$.

Other: $[4,30,31,33,43,50,109,128,138,192,236,330,341,380$, 406].

The term Fréchet-Urysohn space is the usual terminology for a Fréchet space among Russian mathematicians.

An $F U$-space is an abbreviation for Fréchet-Urysohn space.

A space in which each point is $a G_{\delta}-$ set, that is, a countable intersection of open sets, may also be referred to as a space having countable pseudo-character. The term $E_{0}$-space is also in common use for this concept, but the term $G_{8}$-space, which has been used, is unsuitable due to its use for other purposes. Compare: $E_{1}$-space, generalized $G_{\delta}$ space, space of pseudopoint-countable type. Since pseudo-character appears in the Alexandroff and Urysohn memoir of 1929 [2], the following are only some recent references. Conditions for equality of character with pseudo-character: $[1,2,10,166,167,195,239,253$, $267,284,315$ ], relation to other generalizations of first countability: $[37,42,124,141,166,167,195,228,253,267,312,352,353,375,386]$, relation to other cardinality functions: $[32,111,148,186,342]$, relation to paracompactness and other compactness conditions: $[1,2,194,387$, 
388], realcompact: $[164,406]$, function spaces: $[6,144,145,164]$, examples: $[2,6,37,124]$, other: $[139,283,330]$.

The term $G_{\delta}$-space has been used by some mathematicians $[6,164$, 406, p. 163] for a space in which each point is a $G_{\delta}$-set. This term has also been used for at least two other unrelated concepts (spaces in which each closed subset is a $G_{\delta}$-set; spaces which are $G_{\delta}$ subsets of their Stone-Cech compactification).

A (completely regular) space $X$ is a generalized $G_{\delta}$ space iff each point $x$ of $X$ is either an isolated point of $X$ or is such that there exists a real-valued function continuous on $X-\{x\}$ which has no continuous extension to all of $X$. Heider [164] .

A space satisfying the gf-axiom of countability is alternate terminology of Arhangel'skii in [18] for a space satisfying the weak first axiom of countability.

A space is said to have property $H$ iff every accumulation point of a subset is accessible by closed sets. A space with property $H$ is an accessibility space and for regular spaces these concepts coincide. Also compare a $Z$ space. Whyburn $[397,399] /[346]$.

A space $X$ is an $H_{1}$ space iff every countably compact subset is sequentially compact; equivalently, whenever $S$ is a sequence in $X$ having no convergent subsequence, there exists a subsequence $T$ of $\mathrm{S}$ such that the range of $T$ has no accumulation point. Both $\mathrm{H}_{2}$ spaces and $Z$ spaces are $H_{1}$ spaces. Aull [39] and Meyer [254].

A space $\mathrm{X}$ is an $\mathrm{H}_{2}$ space iff whenever $\mathrm{S}$ is a sequence in $\mathrm{X}$ having no convergent subsequence, the range of $S$ has no accumulation point. All sequential spaces and all accumulation complete spaces are $\mathrm{H}_{2}$ spaces. Aull [39].

The terminology k-point has been used with different meanings. The oldest is due to Alexandroff (and Urysohn) in [2] (see also [15, $108,213]$ ). A point $x$ of a space $X$ is a $\kappa$-point iff it is isolated or there exists a sequence of distinct points of $X$ converging to $x$. Tamano in $[374$, p. 230] called a point $x$ of a space $X$ a $k$-point iff whenever $x$ is an accumulation point of a subset $A$ of $X$, there is a compact set $K$ in $X$ such that $x$ is also an accumulation point of $A \cap K$. A space $X$ is a $k^{\prime}$-space iff every point of $X$ is a $k$-point in this sense. For a third meaning, Noble in [301, p. 391] calls a point $x$ of a space $X$ a $k$-point iff for any set $U$ which contains $x$ and such that $U \cup K$ is open in $K$ for every compact subset $K$ of $X$, is itself a neighborhood of $x$. A space $X$ is a $k$-space iff every point of $X$ is a $k$-point in this sense.

A space $X$ is a $k$-space iff every subset of $X$ whose intersection with every compact set $K$ is closed in $K$, is closed in $X$. Definitions vary because the Hausdorff axiom is often assumed. For the origins of 
this important concept see $[8,85]$. A $k$-space is also commonly called a compactly-generated space; less commonly: $K$-space, $k_{3}$-space (by Fuller), Kelley space (by the French), space of class $\mathfrak{\Im}$. Compare de Groot's notion of a $c$-space. See also $k$-point.

General references on $k$-spaces: Arhangel'skii [15] and Steenrod [362].

Textbooks: Kelley [199], Wilansky [406], Willard [408].

Early articles: $[8,135,257,325,94,96,199,274,275]$.

Algebraic topology: $[68,134,360,362,394]$.

Cardinality functions: $[30,34,189,342,343,344]$.

Category: $[123,129,130,134,169,170,172,187,326,327,339,362]$.

Characterizations: $[11,15,17,18,23,96,187,226,244,260,267,274$, $292,351,353,356,362,370]$.

Examples: $[15,48,56,65,77,165,210,263,270,312,331,352,353$, $378,404]$.

Exponential topology: [59, 92, 197, 198, 369] .

Function spaces: $[8,48,75,96,135,145,260,275,300,325,362$, 363,390 ].

General theories: $[121,187,240,286,389]$.

Mappings: $[11,15,16,17,18,19,22,23,24,56,66,68,87,105$, $125,133,146,147,165,178,187,211,226,244,260,261,262,267,274$, 275, 277, 292, 299, 304, 337, 338, 348, 351, 353, 355, 356, 362, 370, $374,377,395,398,409]$.

Metrization: [78].

Paracompactness and normality: [36, 64, 65, 78, 92, 347] .

Products: $[15,22,47,48,59,74,75,92,94,95,97,98,178,179,261$, 262, 275, 299, 301, 304, 307, 308, 309, 362, 363, 365, 374, 376, 378] .

Products of pseudo-compact spaces: [98, 178, 301, 364, 374] .

Realcompactness: $[97,98]$.

Relations to other generalizations of first countability: $[11,15,18$, $19,23,34,44,87,98,132,146,166,189,213,244,261,263,267,270$, $292,312,331,333,338,342,346,352,353,356,375,398,399]$.

Subspaces: $[15,19,23,362,378,392,393]$.

Survey remarks: $[18,30,267,336,339,362]$.

Topological groups: [25, 193, 280, 281, 305].

Other: $[21,43,50,56,57,62,103,130,131,171,210,257,330,404$, $405,412]$.

A subset $A$ of a space $X$ has property $(k)$ iff a subset of $A$ is closed in $A$ whenever it intersects every compact subset $K$ of $X$ in a set closed in $A \cap K$. Compare the concept of a $k$-space. Weddington [393] .

The notation $K$-space is that of $\mathrm{D}$. E. Cohen for a $k$-space.

A space is said to have property $K$ iff every accumulation point of a set is accessible by compact sets. This concept has been called a $k_{1^{-}}$ 
space by Fuller. It is equivalent to a space being hereditarily a $k$-space [23, Proposition 1, and 399], and assuming the Hausdorff axiom, it is also equivalent to the space being a Fréchet space. Halfar [150]/[23, $46,133,224,244,330,399$ ].

A space $X$ is a $k^{\prime}$-space iff whenever a point $x$ is an accumulation point of a set $A$ in $X, x$ is an accumulation point of $A \cap K$ for some compact set $K$. This concept seems to have been discussed originally in 1946 [8]. It has also been called: property $\alpha$ (by Whyburn), $k_{1^{-}}$ space (by Arhangel'skii), and $k_{2}$-space (by Fuller). Compare: $k_{n}$-space, $k$-point. Arhangel'skii $[11,14,15]$ /products: [47], subspaces: [393], relation to paracompactness: $[64,234]$, relation to mappings: $[8,66$, $133,267,353,356]$, other: $[23,34,42,43,121,132,166,187,244,352$, $374,398,399]$.

The terminology $k^{\prime}$-space is also that of Comfort [98] for a $k_{R}$-space.

A space satisfies condition $\left(k_{0}\right)$ iff each sequence having a cluster point has a subsequence which is contained in a compact set. Chiba [88].

A space $X$ is defined to be a $k_{0}{ }^{\prime}$-space by the condition that each sequence having a cluster point $x$ has a subsequence which is contained in a compact set such that $x$ is a cluster point of the subsequence.

The terminology $k_{1}$-space has been used by Arhangel'skii to mean $k^{\prime}$-space (see $k_{n}$-space), and has been used by Fuller to mean a space with property $K$.

The terminology $k_{2}$-space has been used by Arhangel'skii in the sense of the definition given below for a $k_{n}$-space, and has been used by Fuller to mean a $k^{\prime}$-space.

The terminology $k_{3}$-space has been used in the sense below by Arhangel'skii, and has been used by Fuller to mean a $k$-space.

For a positive integer $n$, a space $X$ is a $k_{n}$-space iff for every subset $M$ of $X, \mathrm{Cl}(M)_{k_{n}}=\mathrm{Cl} M$, where $\mathrm{Cl}(M)_{k_{1}}$ is the set of all points $x$ for which there exists a compact set $K$ such that $x \in \operatorname{Cl}(K \cap M)$, and $\mathrm{Cl}(M)_{k_{n+1}}=\mathrm{Cl}\left(\mathrm{Cl}(M)_{k_{n}}\right)_{k_{1}}$. Arhangel'skii $[14,15$, p. 52] .

A space $X$ is a $k_{R}$-space iff every real-valued function on $X$, whose restriction to every compact set is continuous, is continuous on $X$. Complete regularity of the space $X$ is usually assumed. This concept has been called a $k^{\prime}$-space by Comfort in [98]. Michael [263, 270], Comfort [98], Noble $[301,304] /[18$, p. 154, 76, 178, 180, 300, 302, $305,325,390]$.

The terminology Kelley space is that of $[134,326,339]$ and others for a $k$-space.

A space is defined to be a $K F C$-space by the condition that every compact subspace is first countable. Clearly spaces in which every 
compact subspace is metrizable [351, 353], and spaces in which each point is a $G_{8}$-set (by $20 \mathrm{e}$ of Section 3) are $K F C$-spaces. [70] .

An $\mathcal{L}$-space is defined as follows: Let $X$ be a set and $C$ a collection of pairs $(S, x)$ where $S$ is a sequence in $X$ and $x$ is a point in $X$, with $S$ said to converge to $x$, satisfying: (1) if $S$ is a sequence with constant value $x$, then $(S, x) \in C,(2)$ if $(S, x) \in C$ and $R$ is a subsequence of $S$, then $(R, x) \in C$, and (3) if $(S, x) \in C$ and $(S, y) \in C$, then $x=y$. Then $(X, C)$ is an $\mathcal{L}$-space. Notice that $(X, C)$ is not a topological concept and see the discussion of this concept in Section 2. [39, 61, 106, $137,176,208,214,382,400$ ]

An $\mathcal{L}^{*}$-space is an $\mathcal{L}$-space, $(X, C)$ satisfying the additional condition: if $(S, x) \notin C$ then there is a subsequence $R$ of $S$ such that for any subsequence $T$ of $R,(T, x) \notin C$. [101, 106, 137, 138, 208, 214, 241].

The terminology local character is that of [250] for the character of a space.

A space with a locally countable basis is the terminology of McDougle for a first countable space.

Locally separable space is the terminology of Vaidyanathaswamy [382] and Balachandran [51] for a first countable space.

The terminology local weight is that of $[127,187,230]$ for the character of a space.

A space $X$ is maximally resolvable iff it has isolated points or is the union of $\Delta(X)$ disjoint sets each of which intersects each non-empty open subset of $X$ in at least $\Delta(X)$ points, where $\Delta(X)$ is the dispersion character of $X$, i.e., the minimum cardinality of the non-empty open subsets of $X$. All first countable spaces and all regular spaces of point countable type are maximally resolvable. Ceder $[84,85]$, Ceder and Pearson [86], El'kin [113, 114], Pearson [320].

A space is said to be a mosaic space iff it has a cover $\mathscr{K}$ of compact metrizable subspaces and has the (weak) topology: A set $U$ is open iff $U \cap K$ is open in $K$ for every $K \in \mathcal{K}$. Assuming the Hausdorff axiom, the class of sequential spaces and the class of mosaic spaces coincide. Davison [102], Lavallee [224] .

A space $X$ belongs to class $\mathfrak{N}$ iff the projection mapping of $X \times Y$ onto $X$ is a closed mapping for every countably compact space $Y$. Spaces which are $k+S_{4}$ spaces (thus also sequential spaces) belong to $\mathfrak{R}$. Isiwata $[182] /[117,152,212,213,288,345]$.

A space $X$ is $o$-metrizable by an $o$-metric $d$ iff $d$ is a nonnegative real-valued function on $X \times X$ such that $(1) d(x, y)=0$ iff $x=y$, and (2) a subset $F$ of $X$ is closed iff for each point $x$ not in $F, d(x, F)>$ 0 . This concept has also been called generalized metrizable and $g$ metrizable. For $T_{1}$-spaces, $o$-metrizability is equivalent to the space 
satisfying the weak first axiom of countability. All the papers of Nedev in the bibliography are on $o$-metrizability. See also the references for the weak first axiom of countability.

The terminology point character is that of [247] for the character of a space.

A space $X$ is of point countable type or pointwise countable type iff each point is contained in a compact set having countable character in $X$. Compare pseudopoint-countable type. This concept of Arhangel'skii was introduced in [12] and, with proofs, in [15]. Major results may be found in: Arhangel'skii [12, 15, 33], Borges [70], Filippov $[115,116]$, Wicke [401] . Results on mappings: [12, 15, 88, 91, 93, 115, 116, 266, 267, 268, 277, 304, 312, 319, 336, 337, 351, 401,403 , relations to other generalizations of first countability: [12, $15,33,115,116,161,166,167,228,261,312,351,352,376,386]$, cardinality functions: $[28,31,33,342,343,344]$, metrizability: $[12,15$, 70], products: $[12,15,33,304,376], \beta X:[15,386]$, exponential topology: [92, 300], dimension: [319], topological group: [319], survey remarks: $[12,15,18,161,267,277,403]$, other: $[24,27,300$, 353].

Pointwise weight is the terminology of [262] for the character of a space.

The pseudo-character of a set $A$ in a space $X$, denoted by $\Psi(A, X)$, is the least cardinality of a collection of neighborhoods of the set $A$ whose intersection is $A$. The pseudo-character of a space $X$, denoted by $\Psi(X)$, is the supremum of the pseudo-characters of the points of the space $X$. This concept has been discussed in the 1929 memoir of Alexandroff and Urysohn [2]. The terminology pseudo-weight has been used at times in place of pseudo-character. See: space in which each point is a $G_{8}$-set.

A space $X$ is a pseudo-open space iff every quotient mapping onto the space is a pseudo-open mapping. Whyburn defined the concept of an accessibility space and proved that for $T_{1}$-spaces, these concepts coincide. Shirley [346] .

A space $X$ is of pseudopoint-countable type iff each point is contained in a compact set of countable pseudo-character in $X$. Vaughan [386].

The terminology pseudo-weight is that of $[1,139]$ for pseudocharacter.

A space $X$ is said to be a $q$-space iff every point of $x$ is a $q$-point. A point $x$ is a $q$-point iff $x$ has a sequence $\left\{U_{n}\right\}$ of neighborhoods such that if $x_{n} \in U_{n}$ for all $n$, then the sequence $\left\{x_{n}\right\}$ has a cluster point $x^{\prime}$. Assuming regularity, the concept of a $q$-space and a strict $q$ space 
coincide. Assuming paracompactness, the following are equivalent: $q$-space, strict $q$ space, $r$-space, space of point countable type. On mappings: [87, 88, 181, 259, 278, 291, 332, 337] , metrizability: [259, 278], paracompactness: [73, 385], realcompactness: [183, 230], $\beta X$ : [183], products: [376], surveys: [161, 267, 277, 336], other: [70, 166, $173,227,228,261 ; 312,377,403]$.

A space $X$ is a quasi-k space iff every subset whose intersection with every countably compact set $C$ is closed in $C$, is closed in $X$. The concept was introduced by Nagata [292]. On mappings: [54, 146, 181, $213,278,279,292,308,337,355]$, products: [ 308,376$]$, general theory: [54], survey remarks: $[267,277,336]$.

A space $X$ is an $r$-space iff every point $x$ of $X$ is an $r$-point. A point $x$ is an $r$-point iff $x$ has a sequence $\left\{U_{n}\right\}$ of neighborhoods such that if $x_{n} \in U_{n}$ for all $n$, then the sequence $\left\{x_{n}\right\}$ is contained in a compact set. $[234,260,318] /[70,161,166,228,357]$.

A space $X$ is an $r_{0}$-space iff every point $x$ of $X$ has a sequence $\left\{U_{n}\right\}$ of neighborhoods such that if $x_{n} \in U_{n}$ for all $n$, then the sequence $\left\{x_{n}\right\}$ has a subsequence with compact closure. Rishel [337] .

A space of class $\mathbb{S}$ is the terminology of Morita in [274] for a $k$ space.

A space $X$ is an $S_{3}$ space iff every sequentially compact subset is closed in $X$. Aull [39].

A space $X$ is an $S_{4}$ space iff every countably compact subset is closed in $X$. The class of $S_{4}$ spaces includes the classes of sequential spaces and $Z$ spaces. Aull [39]/[102, 376] .

A space $X$ is an $s_{R}$-space iff every real-valued function on $X$, whose restriction to every convergent sequence (with its limit) is continuous, is continuous on $X$; equivalently, every real-valued sequentially continuous function on $X$ is continuous. Noble [304]. Notice that if the words "real-valued" are omitted, this yields a characterization of a sequential space [53]. Vidossich in [388] discusses a similar concept with the words "countable subset" replacing "convergent sequence".

A space $X$ is a sequential space iff every sequentially open set is open. Equivalently, every sequentially closed set is closed. Assuming the Hausdorff axiom, the following are equivalent: (1) sequential space, (2) mosaic space, (3) sequential in the sense of Dudley [106], i.e., a space determined by an $\mathcal{L}^{*}$-space of Fréchet in the sequential manner (as discussed in Section 2). A sequential space has also been referred to as a space determined by sequences, and a sequentially generated space. Closely related to the concept of a sequential space is the concept of a Fréchet space. Section 2 discusses some of the relationships. 
Major references: Franklin [118, 120], Davison [102] .

Early articles: $[61,102,241]$.

Cardinality functions: $[26,30,32,34,249,251,253,254,322,343$, 344].

Category: $[52,169,170,172,187,346]$.

Characterizations: $[34,50,52,61,102,118,146,187,351,352,353$, $370,371]$.

Compact spaces: [30, 32, 102, 122, 239, 254, 285, 287, 329, 380] .

Convergence structure: $[55,61,101,106,138,208,272,400]$.

Examples: $[34,39,41,101,102,117,118,119,120,122,126,188$, $189,192,216,245,324]$.

Function spaces: [101, 102, 106, 245, 246, 248, 251, 273, 300] .

General theory: $[54,121,187,240,286]$.

Mappings: $[53,54,102,118,125,146,168,178,187,212,224,229$, $241,250,267,304,306,307,337,346,351,352,353,370,371,375,377]$.

Modifications of the sequential space concept: $[34,142,157,168,172$, 188, 205, 212, 247, 249, 250, 251, 253, 272, 273, 352, 358] .

Paracompactness and normality: $[36,63,64,215,347]$.

Products: $[62,102,118,119,120,178,212,215,262,267,304,364$, $375,376,377]$.

Relations to other generalizations of first countability: [26, 30, 34, $39,41,102,117,118,120,126,157,175,187,188,224,245,246,247$, $248,249,253,267,273,322,330,331,336,337,341,376,399]$.

Survey remarks: $[245,267,336]$.

Topological group: [25, 329] .

Other: [166, 191, 192, 255, 330, 341, 354, 364, 378, 391] .

A set $F$ is a sequentially closed set in a space $X$ iff every sequence which converges to a point in $X-F$ takes on at most finitely many values in $F$.

Sequentially generated space is the terminology of Franklin and Kohli [125] for a sequential space.

A set $U$ is a sequentially open set in a space $X$ iff every sequence which converges to a point of $U$ is eventually in $U$.

A space $X$ is said to be a singly bi-k space iff whenever a point $x \in \mathrm{Cl} F$ there exists a $k$-sequence $\left\{A_{n}\right\}$ in $X$ such that $x \in \mathrm{Cl}\left(F \cap A_{n}\right)$ for all $n$. Michael [267]/[31, 312, 336, 337, 351, 353] .

The definition of a singly bi-quasi-k space is that of a singly $b i-k$ space with the term " $k$-sequence" replaced by " $q$-sequence". With a slightly different definition, a singly bi-quasi- $k$ space was referred to as property- $(P)$ in [332]. Michael [267]/[278, 312, 332, 336, 337] .

A space $X$ is said to be a strict $q$ space iff every point is contained in a countably compact set of countable character in $X$. Assuming 
regularity, the concept of a strict $q$ space is equivalent to that of a $q$-space. Michael [267].

A space $X$ is said to be a strong accessibility space iff whenever $\left\{A_{n}\right\}$ is a decreasing sequence of subsets of $X$ each having $x$ as a common accumulation point, there exists a closed set $C$ such that $x$ is an accumulation point of $C$ but, for each $n, x$ is not an accumulation point of $C-A_{n}$. Siwiec $[352,353,355]$.

Strongly Fréchet space was the terminology for a countably bisequential space in [352]. This term has also been used in [31, 233] . An abbreviation used in [31] is SFU-space.

A space $X$ is strongly $k^{\prime}$ iff whenever $\left\{A_{n}\right\}$ is a decreasing sequence of sets each having $x$ as a common accumulation point, there exists a compact set $K$ such that $x \in \operatorname{Cl}\left(K \cap A_{n}\right)$ for all $n$. Siwiec [352]/ $[267,336,353]$.

Subsequential is the terminology of Tall [373] for an accumulation complete space.

For the concept of tightness of a space, see: countable tightness.

The terminology very $k$ space is that of Arhangel'skii $[19,23,33]$ for a $k$-space every subspace of which is also a $k$-space. Arhangel'skii showed that for Hausdorff spaces this concept coincides with Fréchet space. Compare: property $K$.

A space $X$ satisfies the weak first axiom of countability iff for each point $x$ of $X$ there exists a countable collection $T_{x}$ of sets $Q(x)$ containing $x$, such that a set $U$ is open in $X$ iff for each $x$ in $U$ there exists a $Q(x) \in T_{x}$ for which $Q(x) \subset U$. This concept is also called the $g f$ axiom of countability. For $T_{1}$-spaces, spaces satisfying the weak first axiom of countability coincide with the $o$-metrizable spaces. Arhangel'skii $[18$, p. 129] $/[79,330,353,354,355]$. Also see the references of Nedev for $o$-metrizability.

A space $X$ is a weakly-k space iff every set whose intersection with every compact set is finite, is closed. Rishel [333, 334], House [174] .

The terminology weakly separable space is that of Whyburn [396] for a first countable space.

A space $X$ is a $Z$ space iff for each point $x$ in $X$ and each subset $M$ of $X$ such that $X$ is an accumulation point of $M$, there exists a subset $P$ of $M$ such that $x$ is the unique accumulation point of $P$. Every Fréchet space is a $Z$ space and every $Z$ space is both an $H_{1}$ space and a space satisfying property $H$. Aull [39], Kannan [191] .

2. A Brief Motivational and Historical Survey. Riesz in 1906, Hausdorff in 1914, and Root in 1914 were the first to consider in one way or another the very natural topological idea of a countable base for the neighborhoods of a point. Though the concept is basic, a more important reason for the interest in spaces satisfying the first axiom 
of countability, or first countable spaces as they are more commonly called today, is that "sequences are adequate". What we mean by this is that a point $x$ is in the closure of a set $A$ iff there exists a sequence in A which converges to $x$. This is certainly a familiar and commonly used property of metric spaces and more generally of first countable spaces. It is clear that a first countable space has this property. However, we may see this in a round-about manner by considering the following condition (which T'ong called condition $c$ ) in [381]): Every point $x$ of the space $X$ has a sequence $\left\{N_{i}\right\}$ of neighborhoods of $x$ with the property that if a point $x_{i}$ is chosen from each $N_{i}$, then the sequence $\left\{x_{i}\right\}$ converges to $x$. The reader may verify that this condition is equivalent to $X$ being first countable, and that the condition implies that sequences are adequate in the sense described above. (This simple property is probably well known, but does not seem to appear in textbooks.)

The reader is probably aware that what we have given as the meaning of "sequences are adequate" is, in fact, the definition of a Fréchet space (in the terminology of American general topologists). Of course, in a first countable space sequences are also adequate in another equally important sense: the sequentially open sets are identically the open sets. This is the defining condition of a sequential space. We have the following implications among these fundamental concepts:

$$
\text { countable } \longrightarrow \text { Fréchet } \longrightarrow \text { sequential. }
$$

Sequential and Fréchet spaces are closely related as the following analogies show. For a sequential space, every sequentially closed set is closed; for a Fréchet space, the sequential closure is the closure. Also, for a sequential space, whenever $A$ is a non-closed set, some accumulation point of $A$ is the limit of a sequence in $A$; for a Fréchet space, whenever $A$ is a non-closed set, every accumulation point of $A$ is the limit of a sequence in $A$. (These conditions actually characterize sequential and Fréchet spaces.)

The idea that sequences are adequate is in the foreground in the definitions given by Fréchet in 1906. The structures which he discussed are not, however, topological spaces as we understand them today. There are two basic ways in which we may translate his $\mathcal{L}$ - and $\mathcal{L}^{*}$-spaces into the current idea of a "topology". (Due to Garrett Birkhoff (with acknowledgment to Baer) [61], and Kisyński [208]. The most useful recent references for topology are Dudley [106], and P. Meyer [250]. See also Kelley's text [199, end of Chapter 2].) Let $X$ 
be a set and $C$ a collection of pairs $(S, x)$ where $S$ is a sequence in $X$, and $x$ is a point in $X$, with $S$ said to be converging to $x$. The collection $C$ is to satisfy the condition that if $(S, x) \in C$ and $S^{\prime}$ is a cofinal (at most finitely many elements have been removed) subsequence of $S$, then $\left(S^{\prime}, x\right) \in \mathcal{C}$. (Fréchet's $\mathcal{L}$ - and $\mathcal{L}^{*}$-spaces assume additional axioms - see Section 1 for definitions of these and other concepts not defined here.) Then a topology $\tau$ may be defined, in the sequential manner, by: a subset $U$ of $X$ is in $\tau$ iff whenever $(S, x) \in C$ and $x \in U$, then $S$ is eventually in $U$. This collection $\tau$, which might also be denoted $\tau(C)$ having been derived from the collection $C$, is easily seen to be a topology and the space $(X, \tau(C))$ is sequential. If $(X, C)$ is an $\mathcal{L}$-space of Fréchet, then $(X, \tau(C))$ is also a $T_{1}$-space (but not necessarily Hausdorff). Conversely, if $(X, \tau)$ is a topological space, then a collection $C=C(\tau)$ may be defined by: $(S, x) \in C$ iff whenever $x \in U \in \tau$, $S$ is eventually in $U$. If $(X, C)$ is an $\mathcal{L}^{*}$-space of Fréchet, then $(X, \tau(C))$ is a $T_{1}$ sequential space and $C(\tau(C))=C$. And if $(X, \tau)$ is a Hausdorff sequential space, then $(X, C(\tau))$ is an $\mathcal{L}^{*}$-space such that $\tau(C(\tau))=\tau$. A collection $\tau^{\prime}$ may also be defined on $X$ in the Fréchet manner, by: a subset $U$ of $X$ is in $\tau^{\prime}$ iff $U=X-\operatorname{Cl}(X-U)$ where a point $x$ is in the closure of a set $F, \mathrm{Cl}(F)$, iff there exists a pair $(S, x) \in$ $C$ such that $S \subset F$. It is well known [217, p. 185] that $\tau^{\prime}$ might not be a topology (the closure operat $>r$ need not be idempotent - though $\left(X, \tau^{\prime}\right)$ is a closure space in the terminology of Cech [82]), but if $\tau^{\prime}$ is a topology, then $\tau^{\prime}=\tau$ and the topology is Fréchet.

Conway [101] points out that if $(X, \tau)$ is the complete metric space $l^{1}$ of all summable (i.e., having finite sum) real sequences (note that $\tau$ is the topology obtained in the sequential manner from $C(\tau)$ ), then there is a non-sequential topology $\tau^{\prime \prime}$ on $X$, which is thus distinct from $\tau$, but for which $C(\tau)=C\left(\tau^{\prime \prime}\right)$. Thus, though a topology uniquely determines the collection of convergent sequences, a collection of convergent sequences determines a "natural" sequential (that obtained in the sequential manner above) topology, but it may not be the only topology having the same collection of convergent sequences. ("Sequences are not adequate" in determining a unique topology.)

The last decade has brought a renewed interest in these spaces from a different viewpoint - that of mappings. Ponomarev [321] and Hanai [153] around 1960 discovered independently that a space is first countable iff it is an open (all mappings are understood to be continuous and onto) image of a metric space; thus yielding a theorem which links a first countable space with the important concepts of a metric space and an open mapping in a particularly interesting manner. Subsequently, Arhangel'skii [13] and Franklin [118] have charac- 
terized Fréchet and sequential spaces as pseudo-open (equivalently, hereditarily quotient) and quotient images of metric spaces, respectively. Bi-sequential and countably bi-sequential spaces have also been defined (see Section 1) and characterized as bi-quotient and countably bi-quotient images of metric spaces, respectively. As a result, the known relations among these mapping concepts immediately yields the following fundamental sequence of implications:

$$
\underset{\text { countable }}{\text { first }} \rightarrow \underset{\text { sequential }}{\text { bi- }} \rightarrow \underset{\text { bi-sequential }}{\text { countably }} \rightarrow \text { Fréchet } \rightarrow \text { sequential. }
$$

It may be of interest to point out that in naming a bi-sequential space, E. Michael [267] chose to use the prefix "bi" to indicate a nice property which this concept shares with first countability. This being that the product of two (or even a countable number of) spaces of this type is again a space of this type. The property of being a bisequential space is also nice because it is hereditary (i.e., it is preserved by arbitrary subsets). Sequential spaces unfortunately have neither of these two properties. The interested reader may consult Michael's paper. (For a survey of the mappings discussed above see Siwiec and Mancuso [356]; for a survey of these and other characterizations of images of spaces, see Michael [267] and Siwiec [353].)

Having discussed images of metric spaces we may point out that an interesting unsolved problem is to characterize closed images and perfect images of first countable spaces. First recall that a perfect image of a metric space is metrizable and that a closed image of a metric space has been characterized as a Fréchet space with an additional condition. (See Lasnev [223], and the discussion of spaces of point countable type and $q$-spaces which follows.) Since a perfect mapping is a bi-quotient mapping, and a bi-quotient image of a bisequential space is again bi-sequential, we have:

$$
\underset{\text { countable }}{\text { first }} \longrightarrow \begin{gathered}
\text { perfect image of a } \\
\text { first countable space }
\end{gathered} \text { bi-sequential. }
$$

Example 1 of Section 4 may be shown to be a perfect image of a first countable space, and of course it is not itself first countable. On the other hand, a slight modification of this example yields an example which is bi-sequential without being a perfect image of any first countable space. Specifically, the example is the one-point compactification of a discrete space of cardinality $\exp \exp \kappa_{0}$. As in example 1, this space is bi-sequential. If it were a perfect image of a first countable (Hausdorff) space $Z$, then $Z$ would be compact because of the 
perfect mapping. However, noting Arhangel'skii's recent discovery [26, see also 322 and 340] of the solution to the well-known problem of Alexandroff and Urysohn, that a space which is compact + first countable ( + Hausdorff) has cardinality less than or equal to the cardinality of the continuum, $c$, we may apply this result to determine that $Z$ has cardinality $\leqq c$. Thus its image, the space with which we are concerned, would have cardinality $\leqq c$, contradicting our assumption. We then have a bi-sequential + compact space which is not a perfect image of any first countable space.

In 1970 Whyburn defined (see Section 1) and characterized an accessibility space as one for which every quotient mapping onto the space is a pseudo-open mapping. Likewise a strong accessibility space has the property that every quotient mapping onto the space is countably bi-quotient. Half of the following is then an immediate corollary: A space is Fréchet (respectively, countably bi-sequential) iff it is sequential + accessibility (respectively, strong accessibility).

Convergent sequences have been central to the discussion thus far. But the set of values of a convergent sequence together with its limit (or one of its limits) forms a compact set, so that not surprisingly, first countable spaces have "nice" properties associated with compact sets. Actually, we have already made use of compactness, indirectly, in our discussion of perfect mappings, and we have stated that a compact first countable (Hausdorff) space has cardinality $\leqq c$. However, the most useful generalization of first countability which deals with compact sets is that of a $k$-space. (Compare de Groot's [140] notion of a $c$-space for non-Hausdorff spaces.) Both a Fréchet space and a sequential space are easily seen to be a $k$-space, but a Fréchet space is actually hereditarily so. The property of being a $k$-space is not hereditary as may be seen by considering a completely regular non- $k$ space and its compactification. In 1966 Arhangel'skii [23 and 19] showed the converse, that is, a (Hausdorff) space which is hereditarily a $k$-space is necessarily a Fréchet space. (This was unexpected because a $k$-space is associated with compact sets and a Fréchet space with convergent sequences. M. Rudin [244 and 399] independently discovered this same result.) The concept of a $k$-space is of use in many other ways; we mention two uses involving mappings: First, every function on a $k$-space $X$, whose restriction to every compact subset of $X$ is continuous, is itself continuous on $X$ (in fact, this characterizes a $k$-space; compare the concept of a $k_{R}$-space). Second, D. E. Cohen [94] characterized a $k$-space as a quotient image of a locally compact space. (This may be compared with a characterization of a sequential space which we have discussed above. See also [267, 353] .) Steenrod 
in [362] has suggested $k$-spaces as a "convenient category" of spaces for algebraic topology, in the sense that it is "large enough to contain all of the particular spaces arising in practice" and has good properties. But the $k$-space assumption is convenient in most of general topology for the same reasons. In particular, one would commonly wish to exclude pseudo-finite spaces (spaces in which every compact subset is finite) from consideration. By making the assumption of a (Hausdorff) $k$-space one does in fact eliminate these. The reader may wish to examine the examples of non- $k$-spaces found in Section 4, of which the most interesting are examples 39 and 69.

Another useful and related idea is that of a $k^{\prime}$-space. (In fact, it was thought at one time that a $k$-space had the $k^{\prime}$-space property; see Arhangel'skii $[15$, p. 31].) hecalling that first countable spaces, even metric spaces, need not be locally compact, we may now consider the following relations:

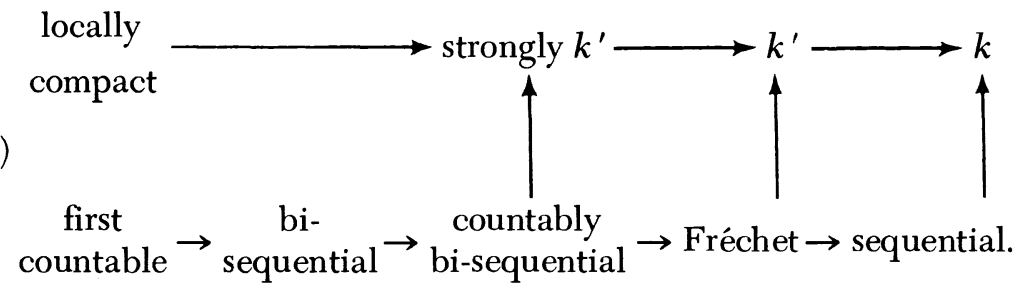

The concepts in this diagram are related to an amazing extent. (i) Locally compact spaces may be defined (namely, characterized) so that the five concepts located in the central region of this diagram and local compactness have definitions which are completely analogous [352]. (ii) The upper sequence of classes of spaces has been characterized by mapping conditions completely analogous to that of the lower sequence (see tables (22) and (23) in Section 3).

Another class of spaces which contains the class of first countable spaces and in whose definition compactness plays a role are Arhangel'skii's spaces of point countable type. This class of spaces also generalizes locally compact Hausdorff spaces, spaces which are perfect preimages of metric spaces, and Cech-complete spaces (completely regular spaces which are $G_{\delta}$ subsets of their Stone-Cech compactification). However, this class is not related to that of the $k^{\prime}$-spaces (examples 4 and 66 in Section 4). These spaces have been especially useful in the study of perfect preimages of metric spaces, known as paracompact $M$-spaces, on which there has come to be an extensive literature. In particular Wicke [401] has characterized spaces of point 
countable type as open images of paracompact $M$-spaces. (Wicke's theorem requires that the image space be a $T_{0}$-space, but Michael [266] has shown that this assumption is not needed. Wicke's theorem is actually true if the words "open mapping" are replaced by "almostopen mapping" or by " $P_{2}$-mapping". For these matters see [353]. See also Chiba [88] and Coban [93, Theorem 14].)

Two other important "mapping theorems" are known for spaces of point countable type. (1) A closed image of a metric space is metrizable if the image is of point countable type. (This result of Arhangel'skii [12] improves a well-known theorem.) (2) Filippov's theorem [115] : a quotient image of a space with a point countable base has a point countable base if the image is of point countable type and if preimages of points are second countable subspaces of the domain. (These results in turn have been improved by Michael - see below.) In regard to a "problem" stated above, Vaughan (and Coban) [386] have pointed out that a perfect image of a first countable space need not be of point countable type nor even of pseudopoint-countable type. (See also [353, p. 135].)

C. J. R. Borges proved a very nice theorem for spaces of point countable type. He showed that if $X$ is the adjunction space of two metrizable spaces, then $X$ is itself metrizable if it is of point countable type. Additionally, he proved a sum theorem for metrizability: If $X$ is of point countable type and if $X$ is dominated (in the sense of Michael) by a collection of metrizable subspaces, then $X$ is metrizable. These and related results appear in [70].

Since a Hausdorff space of point countable type is a $k$-space (this is more difficult to prove than one would expect [15, p. 37]) and since both spaces of point countable type and $k$-spaces have been characterized as images of Hausdorff paracompact $M$-spaces under different mappings (also compare the result of $\mathrm{D}$. Cohen mentioned above), Michael [267] has defined and studied some intermediate classes of spaces, yielding:

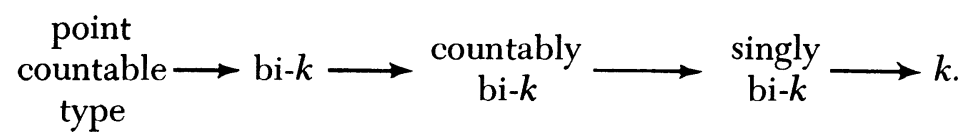

This sequence of concepts also fits directly above diagram (1) given previously with additional implications in an upward direction. (See diagram (2) in Section 3.) Michael [267] has found that the mapping theorems regarding metrizability and point countable base quoted above for the case of point countable type are in fact true assuming only countably bi- $k$ (or even a weaker condition). 
With what we have seen, a question arises whether in fact countably bi- $k$, say, is a "nicer" condition than point countable type. In fact in 1964, almost simultaneously with Arhangel'skii's introduction of spaces of point countable type, Michael introduced $q$-spaces and proved that a closed image of a metric space is metrizable if the image is a $q$-space $[267,259]$. Thus $q$-space is another concept which challenges the importance of point countable type. It appears difficult to determine the relative value of these concepts; however it might be pointed out that in the presence of paracompactness, $q$-spaces and spaces of point countable type are equivalent. Thus the result of Michael concerning the metrizability of a closed image of a metric space is essentially the same as the result of Arhangel'skii quoted before.

(This paragraph is an aside which the reader may omit.) Several other, somewhat related, though less important, concepts have been introduced in efforts to find the weakest conditions needed to prove certain interesting results. Michael's $q$-space is one example, another is condition $\left(k_{0}\right)$, which was introduced as a condition for the countable product of $M$-spaces to again be an $M$-space. Let us pause to examine this more carefully. An $M$-space (respectively paracompact $M$-space) is a quasi-perfect (respectively perfect) preimage of a metric space as we have partially mentioned above. This in itself indicates than an $M$-space is a "nice" concept. However, the product of two $M$ spaces need not be an $M$-space unless the $M$-spaces also satisfy condition $\left(k_{0}\right)$. Thus the question arises whether a "nicer" concept is: $M$-space satisfying condition $\left(k_{0}\right)$. Probably not, but we do have the question here and elsewhere. In particular it is clear from the definitions (which we need not give in this aside) that for spaces satisfying condition $\left(k_{0}\right),(1)$ countably compact $=$ space of class $C^{*}$ of Noble [301], (2) $M$-space = space of class $\mathfrak{E}$ of Ishii, Tsuda, and Kunugi as Chiba [88] points out, and (3) $q$-space $=r_{0}$-space. Note also that (1) implies (2) implies (3), and $r_{0}$-space implies condition $\left(k_{0}\right)$. Now returning to Michael's $q$-space and related concepts we point out to the reader that in Michael's paper [267] the "nicer" concepts seem to be those in our diagram (2) of Section 3 though various weaker properties are also given in his paper which suffice for proving some results (strict $q-q$, point countable type $-r$, countably bi- $k$-property (a) of Michael's Proposition 4.E.5, etc.). Thus again it is not clear which concepts are really "nicer".

Another concept which has been considered for some time, though namelessly until recently, is accumulation complete. This is just the Fréchet property for countable subsets; thus a countable space is 
accumulation complete iff it is Fréchet. But a space need not be countable for this equivalence to hold. Not only will a sequential space do, but a space need only have countable tightness. Thus, if a space has countable tightness and every countable subset is Fréchet, then the space is Fréchet (there are related results stated in Section 3). This and a like condition (spaces determined by countable closed subsets) were first introduced by Moore and Mrowka in 1964 [273] and have been reintroduced in recent years under other names. For Hausdorff spaces we have:

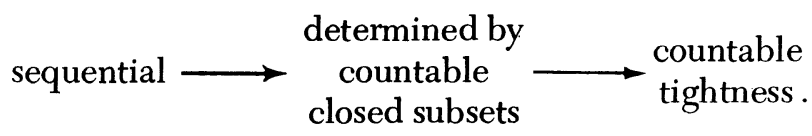

If a space is compact Hausdorff and determined by countable closed subsets, then the space is sequential [273] .

We now state some open problems on this topic. (1) (Franklin) Does Hausdorff + compact + countable tightness imply sequential? (2) (Franklin and Rajagopalan [126]) Does regular + sequentially compact + countable tightness imply sequential? (Compare example 45 and notice that as pointed out in [126], the Proposition of [122] is false and Theorem B is an open problem. However if the answer to (2) is positive then the answer to (1) is also positive for any space of cardinality $<2^{\mathrm{N}_{1}}$, by the Corollary of [122].) (3) (Franklin [122]) Does regular + countable tightness imply determined by countable closed subsets? (Compare example 44). (4) Does Hausdorff + countable tightness (or even a countable space) $+k_{0}$ imply $k$-space? (The answer is positive if $k_{0}$ is replaced by $k_{0}{ }^{\prime}$ in this statement.) (5) For Hausdorff spaces, is hereditarily $k_{0}$ equivalent to accumulation complete? If the answer to (4) is affirmative, then this answer is also affirmative because if $S$ is a sequence with accumulation point $p$ in a hereditarily $k_{0}$ Hausdorff space $X$, then $S \cup\{p\}$ is a Hausdorff $k_{0}$ countable space, thus a $k$-space hereditarily. So that, by the result of Arhangel'skii and Rudin, $S \cup\{p\}$ is Fréchet. Thus there exists a sequence in $S$, and so in $X$, which converges to $p$. (6) Consider a space $X$ having the following property: Whenever a point $x$ is an accumulation point of a set $A$ in $X$ there exists a subset $C$ of $A$ such that $x$ is an accumulation point of $C$ and $C$ has countable closure. This property is clearly "between" the properties of being Fréchet and of being determined by countable closed subsets. Is the property in fact held by spaces which are determined by countable closed subsets? It may easily be seen that a space having property $H$ and having countable tightness satisfies this property. (While this paper was in preprint 
form, V. Kannan has written that he has some solutions to the above problems. In regard to the third problem, he has a regular space with countable tightness which is not determined by countable closed subsets. The example uses elementary measure theory. Regarding the fourth problem he has a countable Hausdorff $k_{0}$ space which is not a $k$ space. And regarding the fifth problem, he has proved the statement affirmatively.)

Considering cardinal numbers other than $\boldsymbol{\kappa}_{0}$ is yet another means of generalizing first countability. We lead into this discussion by first discussing countable pseudo-character. Clearly every $E_{0}$-space (i.e., a space in which each point is a $G_{\delta}$-set) is a $T_{1}$-space, and every regular $E_{0}$-space is an $E_{1}$-space (though there exists a homogeneous Hausdorff $E_{0}$-space which is not an $E_{1}$-space [124]). (Anderson [6] points out that a completely-regular space is an $E_{0}$-space iff each point is a zero-set for some real-valued continuous function.) These two conditions have the advantage of usually being easily verifiable for a given example of a space, and additionally, spaces with a $G_{\delta}$-diagonal (i.e., the diagonal is a $G_{\delta}$-subset of the product of the space with itself) are $E_{0}$-spaces. Since for $E_{0}$-spaces or $E_{1}$-spaces certain pairs of conditions become equivalent - these may be seen in Section 3, but as an example we mention that an $E_{0}$-space of point countable type is first countable - these properties are useful for determining the conditions that particular examples satisfy.

The result just quoted concerning point countable type is interesting - and it also generalizes to higher cardinality. Its history goes back to about the 1920's when Alexandroff and Urysohn in their memoir and also Chittenden [89] proved that a locally compact Hausdorff space is first countable iff each point is a $G_{\delta}$-set. Some of the relations among cardinality concepts are given in Section 3, and we have already stated Arhangel'skii's solution of the Alexandroff and Urysohn problem. However, Arhangel'skii proved more generally, that every Hausdorff space has cardinality strictly less than the Lindelof degree times the character of the space, where the Lindelof degree is the smallest cardinal number $m$ such that every open cover of the space has a subcover of cardinality $m$ or less. (See also Comfort's survey [99].)

Fréchet and sequential spaces, as well as other spaces discussed in this survey, have also been considered with arbitrary cardinality (For example, Meyer has shown that every space having countable tightness is $2^{{ }^{{ }_{0}}}$-Fréchet, and has pointed out that example 51 shows that the converse is false.) These will not be considered in this survey, but the reader may refer to the works of Meyer, and also [26, 28, 30, 34, $127,142,169,186,187,204,272]$. 
As our final consideration we will discuss a space satisfying the weak first axiom of countability whose definition seems to indicate that the concept deserves more attention than it has received. This means of generating a topology does have some interesting properties. (1) Every accumulation complete space which satisfies the weak first axiom of countability is first countable. (2) A space which satisfies the weak first axiom of countability hereditarily is first countable. (See also [354 or 353 IV D].) Means of weakening metrizability by modifying the concept of a metric have also been attempted for some time. A space which has an $o$-metric is in fact equivalent to a space satisfying the weak first axiom of countability. Nedev has done considerable work on this topic from the point of view of $o$-metrizability.

For categorical approaches to the concepts in this survey see: Franklin [123], Herrlich [170], Herrlich and Strecker [172], and Kannan [187] .

3. Some Relations Among the Concepts. For the greater ease of the reader in finding and also in comparing results, we present much of this section in an informal "diagram" form. The statements in (8) and elsewhere are understood in the sense of the following example: If a space $X$ has countable tightness, then $X$ is an accumulation complete space iff $X$ is a Fréchet space. The reader should note that many of the following results are true only with the assumption of the Hausdorff axiom.

A few of the results stated have not appeared in the literature and proofs of these are indicated at the end of the section. There the reader may also find credits for the known results. (Charts (22) and (23) are of interest in this survey because they indicate characterizations of some of the classes of spaces discussed here. However, it does not seem justified to define the large number of mapping concepts given in these charts. The reader interested in the subject may consult the references which are suggested after the charts.) 


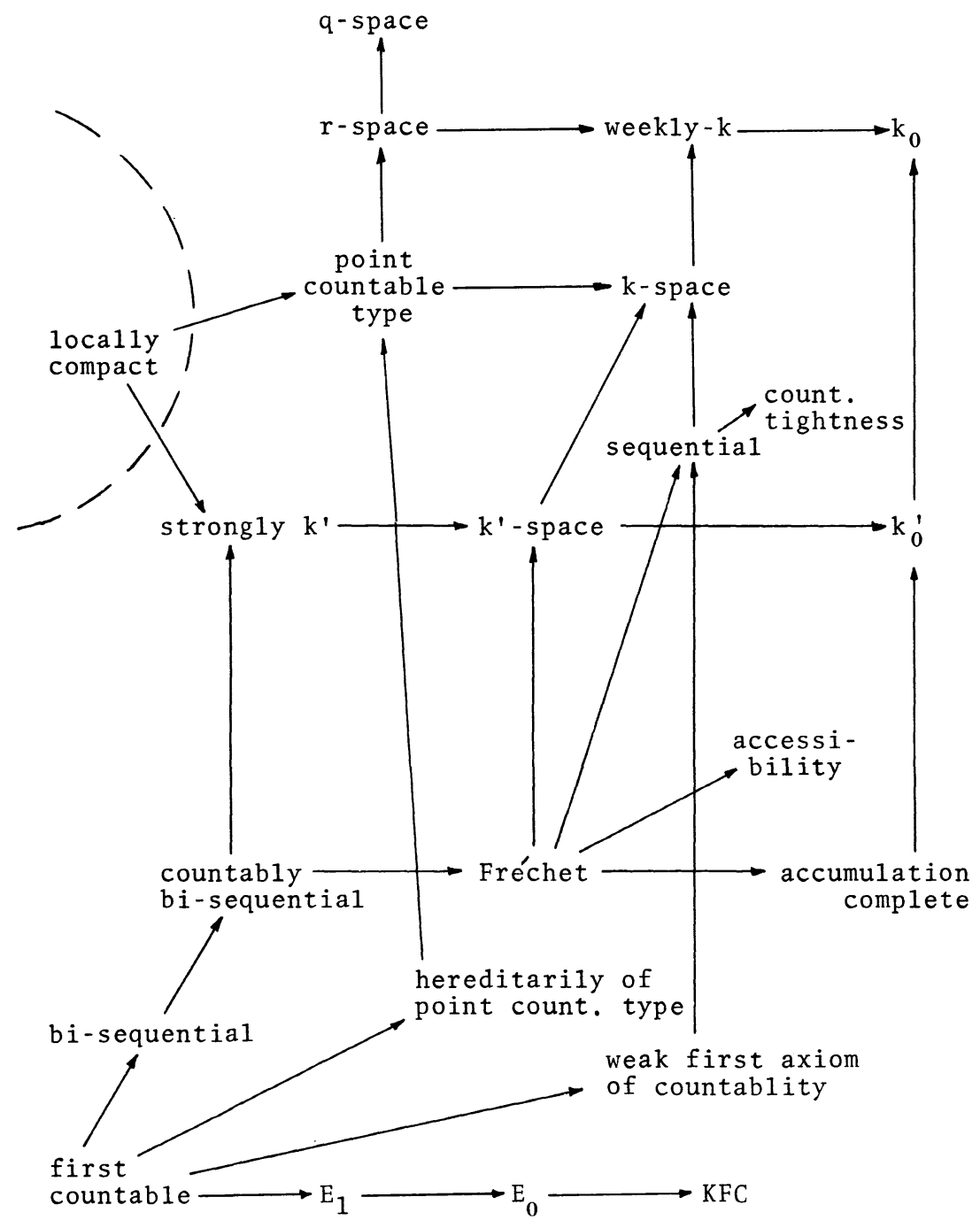

A general diagram. (1) 


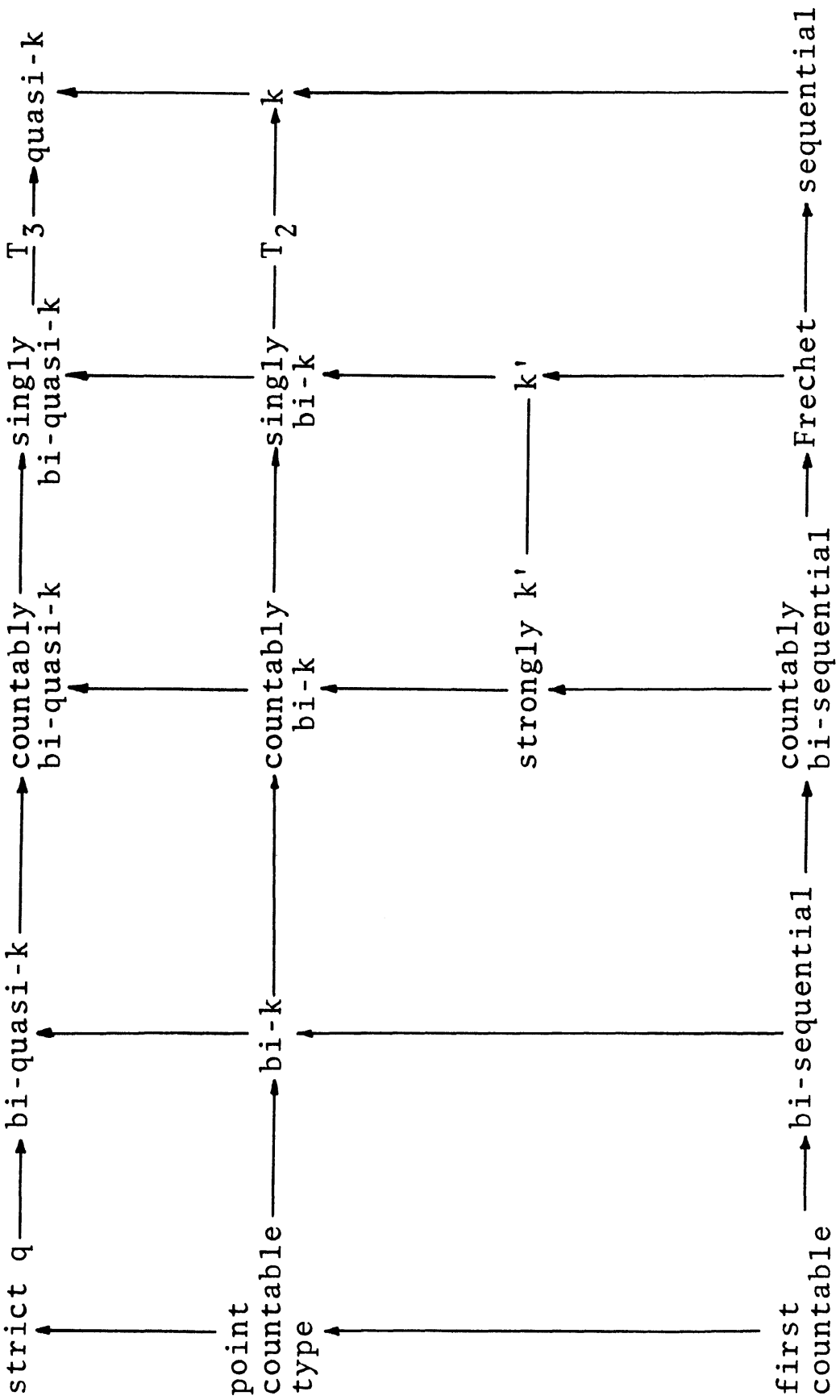

A diagram of $k$ and related spaces. (2) (See (13) and (14) in regard to this diagram.) 


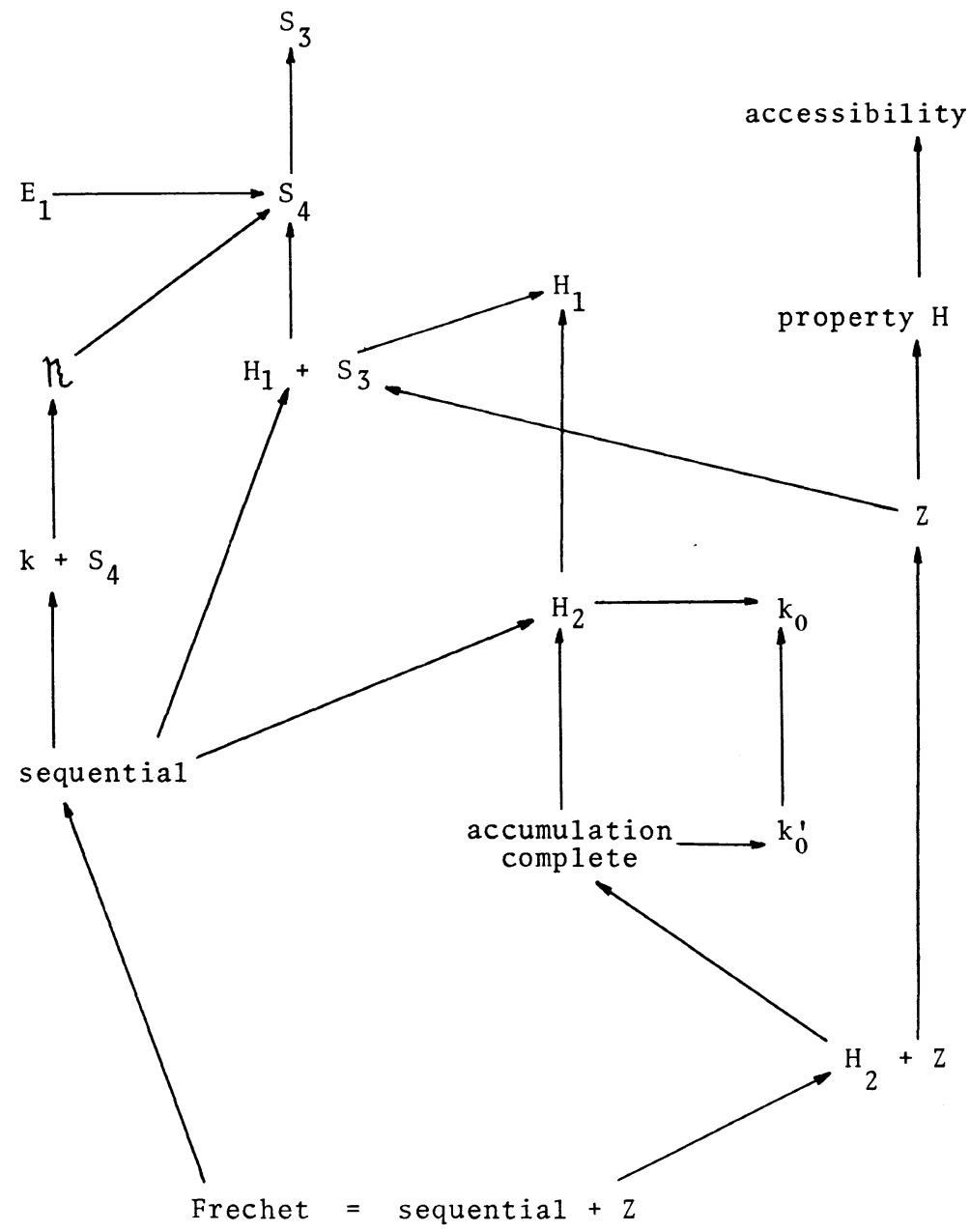

A diagram of concepts defined by sequences (and some related concepts). (4) 


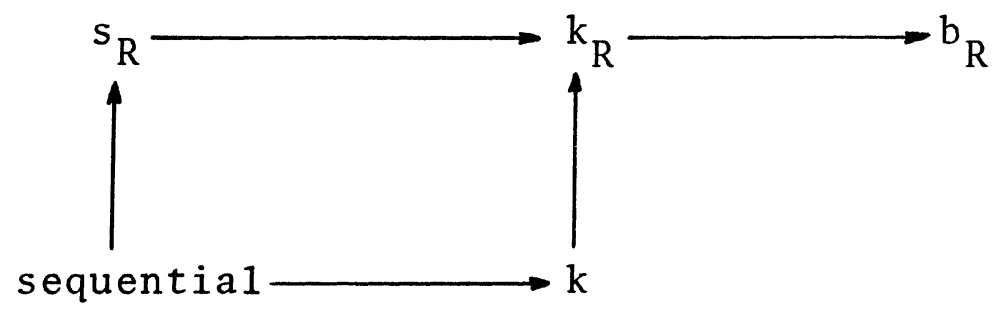

A diagram of concepts defined by functions. (3)

A non-discrete pseudo-finite space (a space in which each compact subset is finite) is not weakly-k (thus also not a $k$-space). (5)

A completely regular space with a non-isolated $P$-point (in the sense of Gillman and Jerison [136]), or a space with a non-isolated point such that every $G_{\delta}$ containing the point is a neighborhood of the point, does not have countable tightness. (6)

If a space $X$ has countable tightness and every countable subspace of $X$ has property $P$, then $X$ also has property $P$, for $P$ being: weakly- $k$, $k^{\prime}$, Fréchet, countably bi-sequential. (7)

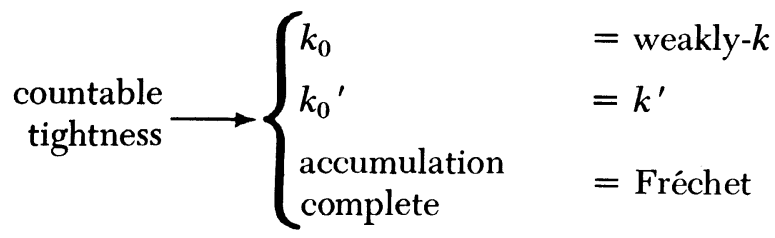

determined by countable closed subsets $+k=$ sequential

hereditarily $k_{0}{ }^{\prime} \quad=$ accumulation complete

hereditarily $k=$ Fréchet

$$
\text { accessibility } \rightarrow \begin{cases}\text { quasi- } k & =\text { singly bi-quasi- } k \\ k_{0}{ }^{\prime}(+ \text { regular })=\text { accumulation complete } & =k^{\prime} \\ k \quad=\text { Fréchet } & \end{cases}
$$




$$
\begin{aligned}
& \begin{array}{l}
\text { strong } \\
\text { accessibility }
\end{array} \\
& \qquad \begin{array}{ll}
k & =\text { strongly } k^{\prime} \\
\text { sequential } & \text { countably } \\
\text { bi-sequential }
\end{array}
\end{aligned}
$$

(See diagram (2) in regard to the above results and also compare $(20 \mathrm{e})$.)

$$
E_{1} \longrightarrow \begin{cases}\text { quasi- } k & =\text { sequential } \\ \text { singly } & \\ \text { bi-quasi- } k & =\text { Fréchet } \\ \text { countably } & =\text { countably } \\ \text { bi-quasi- } k & \text { bi-sequential } \\ \text { bi-quasi- } k & =\text { bi-sequential } \\ q & =\text { first countable } \\ \text { strict } q & =\text { first countable }\end{cases}
$$

(See diagram (2) in regard to the above results.)

The following are equivalent:

(a) Fréchet,

(b) property $K$,

(c) hereditarily a $k$-space,

(d) sequential + accessibility,

(e) sequential $+Z$,

(f) sequential + accumulation complete,

(g) countable tightness + accumulation complete,

(h) countable tightness + hereditarily $k_{0}{ }^{\prime}$,

(i) countable tightness $+k_{0}{ }^{\prime}+$ accessibility (if regular),

(j) $k^{\prime}+$ property $H$,

(k) $k+$ accessibility. 
The following hold:

(a) If $X$ is a space which is sequential but not Fréchet, then $X$ contains a subspace which, with the sequential closure topology (sequentially closed sets are closed), is homeomorphic to example 25 of Section 4.

(b) If $X$ is a space which is Fréchet but not countably bi-sequential, then $X$ contains a subspace which is homeomorphic to example 9 of Section 4 (the sequential fan).

The following are equivalent:

(a) first countable,

(b) for each point $x$ there is a sequence $\left\{U_{i}\right\}$ of neighborhoods of $x$ such that whenever a point $x_{i} \in U_{i}$ for each $i$, the sequence $\left\{x_{i}\right\}$ converges to $x$,

(c) $E_{0}+$ point countable type,

(d) $E_{1}+q$ (or + strict $\left.q\right)$,

(e) accumulation complete + weak first axiom of countability,

(f) accessibility + weak first axiom of countability,

(g) Fréchet + weak first axiom of countability.

If a space is Fréchet + countably bi-quasi- $k+$ regular, then it is countably bi-sequential. Thus a compact Fréchet space is countably bi-sequential. If a space is compact + perfectly normal, then it is first countable.

A space which is countable + regular $+q$ is a separable metrizable space.

For a space $X$, the following relations hold:

(a) $\Psi X \leqq X X \leqq w X$.

(b) $\Psi X \leqq|X|$.

(c) $X X \leqq 2^{\delta X}$, if $X$ is a regular space.

(d) If $K$ is a compact subset of $X, \chi(K, X) \leqq m$, and $\chi(x, K) \leqq m$ for $x \in X$, then $X(x, X) \leqq m$.

(e) $X X=\Psi X$, if $X$ is of point countable type, or if there exists a cover $\left\{K_{\alpha}\right\}$ of compact sets with $X\left(K_{\alpha}, X\right) \leqq \Psi X$. Compare (13).

(f) $|X| \leqq 2^{L X \cdot X X}$. Thus, if $X$ is compact + first countable, then $|X| \leqq c$.

(In the above, $|X|$ is the cardinality of $X, X X$ is the character of $X$, $\delta X$ is the density of $X$ (i.e., the least cardinality of a dense subset of $X$ ), $L X$ is the Lindelof degree of $X$ (i.e., the least cardinal number $m$ such that every open cover of $X$ has a subcover of cardinality $m$ or less), $\Psi X$ is the pseudo-character of $X$, and $w X$ is the weight of $X$ (i.e., the least cardinality of an open base for $X$ ). See Comfort [99] for an excellent survey of cardinality concepts.) 
For a completely regular space $X, \chi(x, X)=\chi(x, \beta X)$ for every $x$ in $X ; \chi(x, \beta X)$ is uncountable for every point $x$ of $\beta X-X$.

\begin{tabular}{|c|c|c|c|c|}
\hline & quotient & $\begin{array}{r}\text { pseudo- } \\
\text { open }\end{array}$ & \multirow{2}{*}{$\begin{array}{l}\text { countably } \\
\text { bi-quotient } \\
\text { strong } \\
\text { accessi- } \\
\text { bility }\end{array}$} & \multirow[t]{2}{*}{ bi-quotient } \\
\hline quotient & $\cdots-$ & $\begin{array}{l}\text { accessi- } \\
\text { bility }\end{array}$ & & \\
\hline $\begin{array}{r}\text { pseudo- } \\
\text { open }\end{array}$ & --- & --- & & \\
\hline $\begin{array}{l}\text { compact - } \\
\text { covering }\end{array}$ & $\mathrm{k}$ & $k^{\prime}$ & strong1y $\mathrm{k}^{\prime}$ & $\begin{array}{l}\text { local1y } \\
\text { compact }\end{array}$ \\
\hline $\begin{array}{l}\mathrm{km}- \\
\text { covering }\end{array}$ & sequential & Frechet & $\begin{array}{l}\text { countably } \\
\text { bi-sequent. }\end{array}$ & $\begin{array}{l}\text { 1oc. compact } \\
+ \text { loc.metriz }\end{array}$ \\
\hline $\begin{array}{l}\text { sequence- } \\
\text { covering }\end{array}$ & sequential & Frechet & $\begin{array}{l}\text { countably } \\
\text { bi-sequent. }\end{array}$ & \\
\hline $\begin{array}{c}\text { sequentially } \\
\text { quotient }\end{array}$ & sequential & Frechet & $\begin{array}{l}\text { countably } \\
\text { bi-sequent. }\end{array}$ & \\
\hline $\begin{array}{l}\text { countable } \\
\text { covering }\end{array}$ & $\begin{array}{l}\text { countable } \\
\text { tightness }\end{array}$ & $\begin{array}{l}\text { countable } \\
\text { tightness }\end{array}$ & & \\
\hline
\end{tabular}

Characterizations of spaces $X$ by the property that every $\cdots$ (left column) $\cdots$ mapping onto $X$ is a $\cdots$ (top row) $\cdots$ mapping. 


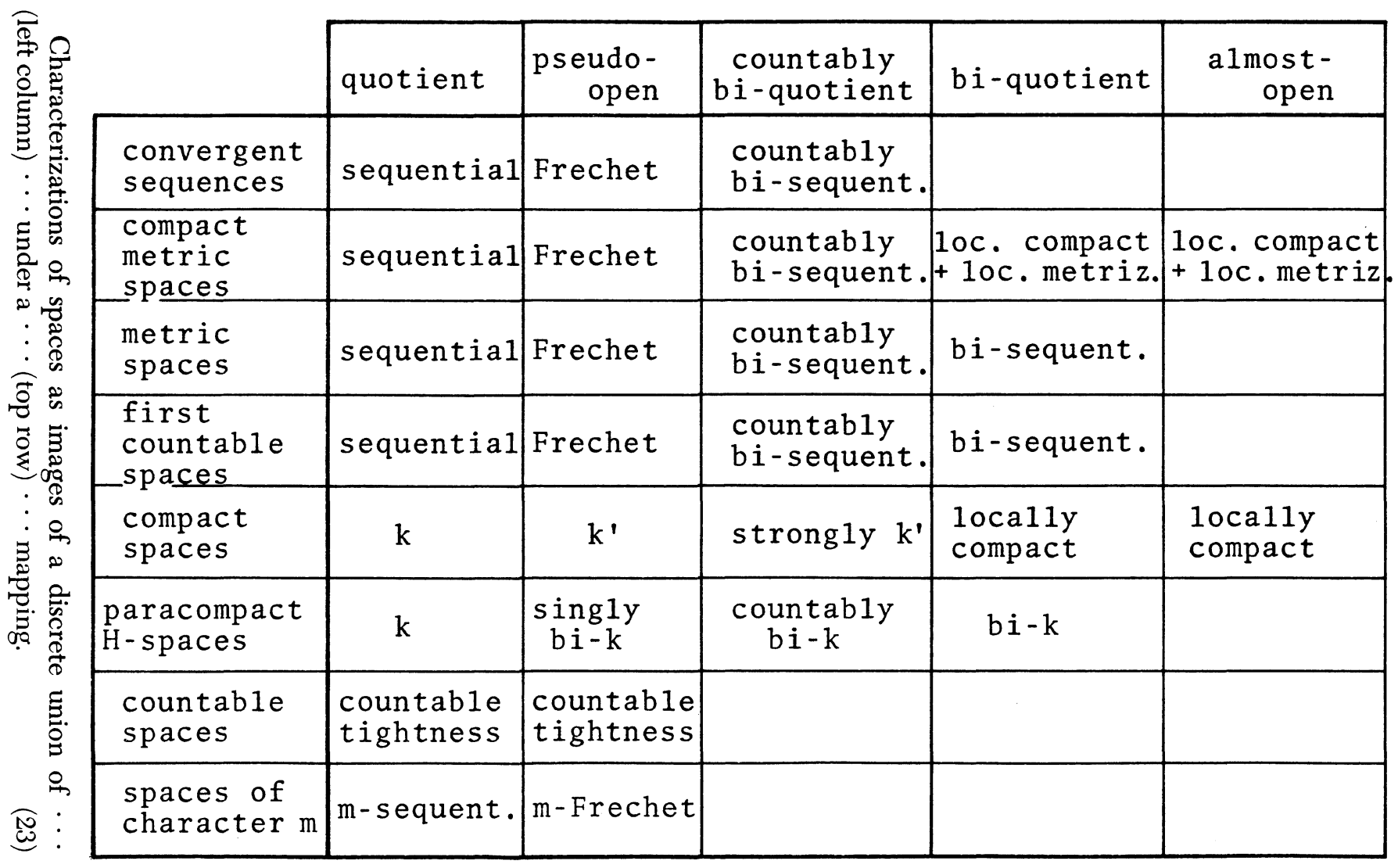


Credits and proofs for the above results.

Diagram (2) is taken from Michael [267]; most of diagram (4) is from Aull [39]; the fact that a space which is $k+S_{4}$ belongs to $\mathfrak{R}$, in diagram (4), is similar to a result of Isiwata [182, Theorem 1.3] which the reader may verify by modifying Isiwata's proof. (Isiwata has more on this last subject. See also Scarborough [345, Corollary 5], Nadler [288], Hanai [152, Theorem 3], and Noble [302, 303, 307]. A partial converse of Hanai [152, Theorem 4] may be improved somewhat to say: If $X$ is a $T_{1}$ non-discrete space having countable tightness and $Y$ is a space such that the projection of $X \times Y$ onto $X$ is closed, then $Y$ is countably compact. A related result is also in [345, Corollary 5 , and 288].)

Result (5) is easily proved and may be left to the reader. Statement (6) is due to Paul Meyer.

The Fréchet and countably bi-sequential cases of (7) and (8) are proved by Michael in [267]. Noting that for countable spaces: $k_{0}=$ weakly $-k$ and $k_{0}{ }^{\prime}=k^{\prime}$, the proofs of the other cases are trivial.

Result (9) is due to Kannan [187]. The second statement of (10) is a well-known result of Arhangel'skii [23] and independently, of Mary Ellen Rudin (see [399] and [244]), while the first statement of (10) is an immediate corollary.

It is easily proved that a regular + accessibility $+k_{0}{ }^{\prime}$-space is accumulation complete. The other parts of (11) and (12) follow immediately from the mapping characterizations of the spaces given in charts (22) and (23).

The results of (13) and (14) with the $E_{0}$-space and $E_{1}$-space assumptions have been discussed by several researchers; see Michael [267 Section 7] in particular. The writer showed in [351 or 353] that the $E_{0}$ space assumption could be replaced by the assumption of a KMspace (i.e., a space in which every compact subspace is metrizable). Here we show a better result, that KFC-space suffices. (Notice that every $E_{0}$-space is a KFC-space by $(20 \mathrm{e})$.) The cases of a $k_{0}{ }^{\prime}$-space, $k$-space, and space of point countable type easily follow directly from the definitions and $(20 \mathrm{~d})$. The three "bi- $k$ " cases may be proved directly or in the manner of the following: If $X$ is a space which is bi- $k$ and KFC then, by results given in Michael [267, Theorem 3.E.3, etc.], the space $X$ is a bi-quotient image of a paracompact $M$-space $Z$ which is a subspace of $X \times M$ for some metric space $M$. Then $Z$ is a KFCspace because if $K$ is compact in $Z$, then its projection $\pi_{1}(K)$ into $X$ is compact in $X$. Since $X$ was assumed to be $\mathrm{KFC}, \pi_{1}(K) \times M$ is first countable, so that the subspace $K$ is also first countable. But a paracompact $M$-space is known to be of point countable type [267], so 
that $Z$ which is $\mathrm{KFC}$ and of point countable type is first countable by what we have mentioned above. The space $X$ being a bi-quotient image of the first countable space $Z$ is then bi-sequential [267]. The two remaining cases of (13) are now trivial. Notice that the results of (14) expect for the $q$-space case may be generalized in a like manner. In particular the $E_{1}$-space assumption may be replaced by the assumption that every countably compact subspace is first countable.

The equivalence of Fréchet to (b) and (c) of (15) is (10) above. The equivalence to (d) is a repeat of (11), (e) is due to Aull [39], (f) is due to Howes and Chandler [175] , $(\mathrm{g})$ is a repeat from (8), (h) follows from (10), while (i) follows from (11). The last two conditions are due to Shirley [346] and Aull [44]. Result (a) of (16) is due to Franklin (see example 25 of Section 4), while (b) is a variation of some yet unpublished work of P. Harley and K. van Doren dealing with metrizability. We will prove this result here. Thus, let $X$ be a space which is Hausdorff, Fréchet, but not countably bi-sequential. We wish to show that $X$ contains a subspace homeomorphic to example 9 . Since $X$ is not countably bi-sequential, there exists a decreasing sequence $\left\{A_{n}\right\}$ of sets having a common accumulation point $x_{0}$ and such that no sequence $\left\{x_{n}\right\}$ with $x_{n} \in A_{n}$ for all $n$, can converge to $x_{0}$. Let $\left\{x_{n}{ }^{1}\right\}$ be a sequence of distinct points of $A_{1}$ converging to $x_{0}$. There exists an $i_{2} \in N$ such that $i_{2}>1$ and no subsequence of $\left\{x_{n}{ }^{1}\right\}$, which is contained in $A_{i_{2}}$, can converge to $x_{0}$. Let $\left\{x_{n}{ }^{2}\right\}$ be a sequence of distinct points of $A_{i_{2}}$ converging to $x_{0}$. Thus $\left\{x_{n}{ }^{2}\right\}$ may be assumed to have no points in common with the sequence $\left\{x_{n}{ }^{1}\right\}$. There exists an $i_{3} \in N$ such that $i_{3}>i_{2}$ and no subsequence of $\left\{x_{n}{ }^{1}\right\}$ and $\left\{x_{n}{ }^{2}\right\}$, which is contained in $A_{i_{3}}$, can converge to $x_{0}$. Continuing this procedure, we obtain $x_{n}{ }^{j}$, and we may set $X^{\prime}=\left\{x_{0}, x_{n}{ }^{j} \mid j, n \in N\right\}$. Then for each $j$, there are at most finitely many points $x_{n}{ }^{j}$ which are not isolated in $X^{\prime}$. For the proof of this, suppose there is a $j \in N$ for which there are infinitely many $x_{n}^{j}$ not isolated in $X^{\prime}$. Denote these by $D$. Then each $x_{n}{ }^{j}$ of $D$ is an accumulation point of $X^{\prime}$. Since $X^{\prime}$ is Frechet, there is a sequence $S_{j n}$ in $X^{\prime}$ which converges to $x_{n}{ }^{j}$. Clearly $S_{j n}$ has only finitely many points in common with each sequence $\left\{x_{m}{ }^{k} \mid m \in N\right\}$. We may assume that $S_{j n}$ was chosen to be contained in $\left\{x_{m}{ }^{k} \mid k \geqq n, m \in N\right\}$. Let $S$ be the union of all these $S_{j n}\left(\right.$ for $x_{n}{ }^{j} \in D$ ), with $\left\{x_{0}\right\} \cup D$ removed from $S$. Then $x_{0}$ is an accumulation point of $S$. Since $S$ is Fréchet, there is a sequence in $S$ which converges to $x_{0}$. But then this sequence has only finitely many points in common with each sequence $\left\{x_{m}{ }^{k} \mid m \in N\right\}$. Let $S^{\prime}$ be a subsequence having at most one point in common with each $\left\{x_{m}{ }^{k} \mid m \in N\right\}$. Let $S^{\prime \prime}$ be $S^{\prime}$ with a repetition of terms, if necessary, so that $S^{\prime \prime}$ has an element $y_{n}$ of each $A_{n}$ with $S^{\prime \prime}=\left\{y_{n} \mid n \in N\right\}$. 
Then $y_{n} \rightarrow x_{0}$ which contradicts the original assumption. Thus, for each $j$ there are at most finitely many points $x_{n}{ }^{j}$ not isolated in $X^{\prime}$. Let $X^{\prime \prime}$ consist of the isolated points of $X^{\prime}$ union $\left\{x_{0}\right\}$. Then $X^{\prime \prime}$ has $x_{0}$ as its only non isolated point. To show that $X^{\prime}$ is example 9 , let $G$ be an arbitrary open neighborhood of $x_{0}$ in the topology of example 9 . Then $X^{\prime \prime}-G$ is of the form $\cup\left\{D_{j} \mid j \in N\right\}$, where each $D_{j} \subset$ $\left\{x_{n}{ }^{j} \mid n \in N\right\}$. By Theorem 5.1 of Olson [312], since each $D_{j}$ is closed, their union is closed in $X$, and so also closed in $X^{\prime \prime}$. Thus $G$ is open in $X^{\prime \prime}$.

A countable regular space is easily seen to be an $E_{1}$-space, thus (19) follows from (17d). Results (d) and (e) of (20) are due to Coban [91, 90, p. 143], while (f) is due to Arhangel'skii [26] (see also [28, 99, $322,340])$. The results of (21) may be found in Wilansky [406, p. 151 \#209 and p. 195 \#112].

Chart (22) may be found in [352, 356, and 399]. In chart (23) almost all the spaces $X$ characterized have the property that the discrete union referred to consists of the discrete union of all subspaces of $X$ of the stated type. The "sequential" entries are due to Franklin [118], the "Fréchet" entries to Arhangel'skii [13], the "countably bi-sequential" entries to Siwiec [352]. The "bi-quotient" column is due in whole or in part to Michael [267] and Morita [274]. In the "compact" row the first entry is due to Morita [274], the second to Arhangel'skii [15], and the third to Siwiec [352]. The "paracompact $M$-space" row is due to Michael [267], the following row to Rishel [331] and Kannan [187]. The chart appearing here is very similar to a chart of Kannan [187, p. 164] and a chart of Michael [267] to which the reader might also refer. Charts (22) and (23) are taken from [353].

4. The Examples. In this section we present a number of examples of Hausdorff, non-first countable spaces gathered from the literature. The properties which the following examples satisfy are given in a chart which follows. Some good references for additional examples are Franklin [118 and 120], Franklin and Rajagopalan [126], Michael [267], and Steen and Seebach [361] .

1. The one-point compactification, $R^{*}$, of $R$, where $R$ is the set of real numbers with the discrete topology [361, \#24]. By Michael [267, Example 10.15] this space is bi-sequential. It is in fact a perfect image of a first countable space (the space given in Bourbaki [72, Exercise 2.13d] ).

2. Let $Z$ be the unit square with lexicographic order and the order topology [361, \#48], let $Y=Z \times Z, K$ the diagonal in $Y$, and $X=$ $Y / K$. (The space $Y / K$ is the quotient image of $Y$ under the mapping 
$f$ which identifies $K$ to a point. In other words, $Y / K=(Y-K), U$ $\{p\}$ where $p$ is a point not in $Y-K, f: Y \rightarrow Y / K$ is defined by $f(x)=x$ for all $x$ in $Y-K$ while $f(x)=p$ for $x$ in $K$, and $Y / K$ has the topology obtained by requiring $f$ to be a quotient mapping.) Then $X$ is a perfect image of the space $Y$ (a compact first countable space), and $X$ is not first countable because $K$ is not a $G_{\delta}$-set in $Y$. [267, Example 10.4]

3. The ordinal space $\left[0, \omega_{1}\right)$, where $\omega_{1}$ is the first uncountable ordinal, with the set of countable limit ordinals identified to a point (see example 2 above for "identified to a point"). This is $Y$ of example 3.3 in [259].

4. The space $X / S$ described in remark 3.3 of Borges [69] .

5. The space $X / K$ where $K$ is the closed unit interval in the $x$-axis and $X$ is the example of remark (2) on page 105 of Heath [160].

6 . The space $X / K$ where $K$ is the closed unit interval in the $x$-axis and $X$ is the space of example 12.1 of Michael [260], [267, Example 10.3].

7. The plane with the topology generated by sets of the form $U$ where $U$ is an open interval of a line through the origin such that the interval does not contain the origin, or $U$ is the union of the collection of open intervals each of which contains the origin and such that each line through the origin contains one of these open intervals. Example C of Bing [60, or 361 \# 141]. Compare example 13.

8. The one-point compactification of a discrete space whose cardinality is a measurable cardinal. Michael [267, Example 10.13] .

9. A countable space $X$ of Arens [9, the space $X$ on p. 233] commonly called the "sequential fan". Let $X_{n}=\{0,1,1 / 2, \cdots\}$ with the usual topology for each $n \in N$. Consider the discrete union of these $X_{n}$, and let $X$ be the quotient obtained by identifying the zeros to a point. See also [15, page 25 Example 2.3, 126, page 311, 252]. This is a subspace of example 16 .

10. The second example on page 475 of Duda [ 104] .

11. The space of rational numbers with the set of integers identified to a point [118, example 1.11] .

12. The space of real numbers with the set of integers identified to a point.

13. The plane with the topology generated by sets of the form $U$ where $U$ is an open interval of a line through the origin such that the interval does not contain the origin, or $U$ is an open sphere centered at the origin with a finite number of open radii removed. Anderson [6, example 3]. Compare example 7.

14. The plane with the $x$-axis identified to a point [199, example 3.R] . 
15. The first example on page 475 of Duda [104] .

16. The discrete union of a countable number of copies of the closed unit interval, with the point zero in each of the copies identified to one point. This space contains example 9 as a subspace.

17. Okuyama's image space in section 4 of [311].

18. The countable, zero-dimensional, homogeneous space of Franklin and Rajagopalan [126, example 3.1] .

19. The zero-dimensional topological group of Franklin and Rajagopalan [126, example 2.1] .

20. The discrete union of $2^{\boldsymbol{x}_{0}}$ copies of example 1 , with the point at infinity of each copy identified to one point [265, p. 17] .

21. The one-point compactification of the space $\Psi$ of Isbell. Franklin [120, example 7.1] .

22. The real numbers provided with the topology generated by the union of the usual topology and all sets of the form $U \cup\{0\}$, where $U$ is a usual open neighborhood of the sequence $\{1 / n\}$. Franklin [118, example 1.8].

23. Let $X$ be the real line, let $f(x)=x$ if $x$ is not a positive integer, and let $f(x)=-1 / x$ otherwise. The image space, with the quotient topology, is the example of T'ong [381, example 1, or 18, example $2.2]$.

24. Ceder's example 9.3 in [83] .

25. This is a countable space due to Arens [9, Y on page 233] or see Franklin [120, example 5.1]. Franklin has shown that every space which is sequential but not Fréchet contains a subspace which, with the sequential closure topology, is homeomorphic to this example. (In the sequential closure topology every sequentially closed set is closed.) The space $X=(N \times N) \cup N \cup\{0\}$ with each point of $N \times N$ an isolated point. A basis of neighborhoods of $n \in N$ consists of all sets of the form $\{n\} \cup\left\{(m, n) \mid m \geqq m_{0}\right\}$. And $U$ is a neighborhood of 0 iff $0 \in U$ and $U$ is a neighborhood of all but finitely many $n \in N$. Example 38 is the subspace obtained by deleting $N$ from $X$.

26. The radial topology of the plane: a set $U$ is open iff for each point $p$ of $U, U$ contains a line segment through $p$ in each direction. The space is separable, but not Lindelöf and not regular. [410]. Compare example 28.

27. The product space of the space of example 12 with a closed unit interval. Franklin [120, example 7.4] .

28. The Archimedean topology of the plane: a set is open iff its intersection with each horizontal line and each vertical line is a set open in the usual topology of the line [15, page 30 and page 58; also 256]. Compare example 26. 
29. The space $X_{1}$ of example 3.2 on page 35 of [15] .

30. The countable, zero-dimensional space $S_{\omega}$ of Arhangel'skii and Franklin [34], which has no point of first countability.

31. An example of Kofner [209, example 2, or 210, example (9.2)].

32. A countable example of Kofner [209, example 3]. The product of this space with itself is not a $k$-space.

33. Example 4 of Kofner [209].

34. An example of Arhangel'skii [18, p. 149, example 5.1, or equivalently, 20, p. 1260].

35. The product space of the real line with example 11. Michael [260, example 12.6] .

36. The product space of the space of example 16 and a closed unit interval. Michael [267, example 10.2] .

37. A countable, connected, nowhere first countable example of Kannan [189, Theorem 3] .

38. The countable pseudo-finite (every compact subset is finite) example of Arens. [ 9 the space $Z$ on page 234, or 199, Problem 2.E]. See example 25.

39. The subspace $N \cup\{p\}$ of $\beta N$ where $p$ is any point of $\beta N-N$. This space is pseudo-finite, not a subspace of any sequential space [117], and is not of class $\Re$ [212]. See: [136, Problem 6.E.5, or 260, example 12.5].

40. The product space of the space of rational numbers with the space of example 11. Franklin [118, example 1.11, or 260, example 12.6].

41. A countable space of Arhangel'skii identified as example $L$ in [15, p. 15, example 2.2]

42. A countable, zero-dimensional example of Appert [361, \#98] .

43. A countable space of Arhangel'skii identified as example $\pi$ in $[15$, p. 57 , example 3.5]. No point of the space is a $\boldsymbol{\kappa}$-point (in the sense of Alexandroff and Urysohn), in fact the space is pseudo-finite.

44. The space is $\beta N$ retopologized with a topology generated by the union of the usual topology for $\boldsymbol{\beta} N$ and the family $\{N \cup\{p\} \mid p \in \beta N$ $-N$ \}. Franklin [122, Theorem A]. Example 55 is $\beta N$ with its usual topology.

45. Example 1.2 of Franklin and Rajagopalan [126].

46. An example of Heath [ 162 or 163] .

47. The product space of the space of rational numbers with the space of example 16 [267, Example 10.1] .

48. An example of Aull [37, example 1]. This is not an $E_{1}$-space though it is an $E_{0}$-space. 
49. The subspace of $\left[\mathbf{0}, \omega_{1}\right]$, where $\omega_{1}$ is the first uncountable ordinal, obtained by removing the set of countable limit ordinals [165, example $\mathrm{H}$ on p. 85]. This example of a pseudo-finite space is very similar to the following example.

50. The set of real numbers with an additional point "at infinity". A set is closed if it contains the point at infinity or if the set is countable. This example of Gál [39, example 5 or 352, Problem 2.8b] and the preceding example are very similar.

51 . The set of ordinal numbers less than or equal to the first uncountable ordinal with the usual order topology [361, \#43] .

52. The Tychonoff plank in the version with the corner point $\left(\omega_{1}, \omega_{0}\right)$ included [361, \#86]. Compare example 60.

53. The product of $2^{\mathrm{K}_{0}}$ copies of the closed unit interval [ 361 , $\# 105,197,198]$. Priestley shows that this space is badly non-Fréchet since it has a countable dense subset that contains no nontrivial convergent sequences $[323,324]$.

54. The product of $2^{K_{0}}$ copies of a discrete two point space.

55. The Stone-Cech compactification $\beta N$ of the countable discrete space $N$ [361, \#111, or 136, example 6.10]. This example is not an $H_{1}$ space [39] and does not belong to class $\mathfrak{N}$ [182, example 2.3] . For the character of this space see [149]. Example 44 is a modification of this example.

56. The Stone-Cech compactification $\beta Q$ of the space of rational numbers [136, example 6.10]. $\beta Q$ does not belong to $\mathfrak{N}$ [182, example 2.3] .

57. The Stone-Cech compactification $\beta R$ of the space of real numbers [136, example 6.10] $\beta R$ does not belong to $\mathfrak{N}$ by [182, example 2.3].

58. Example 1.1 of Franklin and Rajagopalan [126] .

59. The Stone-Cech compactification of example 50. This is the space $Y$ of [267, Example 10.5] .

60. The Tychonoff plank in the version with the corner point $\left(\omega_{1}, \omega_{0}\right)$ deleted [136, example 8.20, or 257, \#87]. Compare example 52.

61. The space $X$ in section 4 of Morita [276] .

62. The space $Y$ in section 4 of Morita [276].

63. The product space of $\left[0, \omega_{1}\right)$ with $\left[0, \omega_{1}\right] .[199$, Problem 4.E, or 165, Example 2.3] .

64. Example X of Suzuki [372] with $M_{\alpha}=\left[0, \omega_{1}\right]$ and cardinality of $A=\boldsymbol{\kappa}_{1}$.

65. The real line with an open base consisting of the usual open intervals with at most a countable set of points deleted. This is a pseudo-finite example of Alexandroff and Urysohn [2, example 2] . 
66. Example 10.5 of Michael [267].

67. The space $X \times Y$, where $X=\{0,1,1 / 2, \cdots\}$ with the usual topology and $Y$ is example 9 (the sequential fan). Bagley and Weddington $[47$, Theorem 1].

68. The example $P \cup N$ of Novak [361, \#112, or 136, example 9.15].

69. The product space of $2{ }^{\kappa_{0}}$ copies of the real line.

70. The modification of Bing's example $G$ by Michael in [258, example 2] .

71. Isiwata's modification ([183], or the space $X$ of [267] Example 10.7) of Novak's example, $P \cup N$, number 68 above.

72. Isiwata's modification of Novak's product space (the space $X \times Y$ of [267] Example 10.7).

In the chart which follows, the second column refers to the separation axioms which the examples satisfy - note that all examples are Hausdorf - with "P" meaning paracompact Hausdorff, "K" meaning compact Hausdorff, and "RC" meaning regular and countable. An " $\mathrm{H}$ " means that the example has the stated property hereditarily, " + " means the example has the property, but some subspace does not have the property, " - " means the example has the property, and "-" means the example does not have the property. Blanks in the chart indicate cases which have not been answered. The letter "c" denotes the cardinality of the continuum. However, the continuum hypothesis is at times assumed, so that in some cases "c" actually denotes that the cardinality, $m$, satisfies $\boldsymbol{\aleph}_{0}<m \leqq c$. 


\begin{tabular}{|c|c|c|c|c|c|c|c|c|}
\hline & & 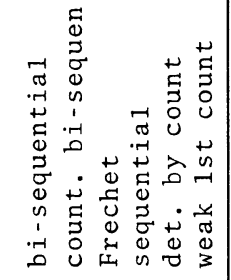 & 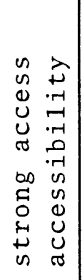 & 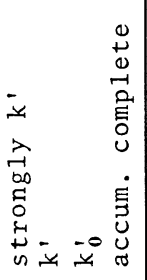 & 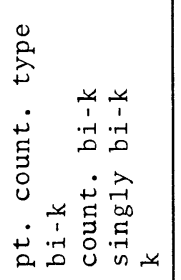 & 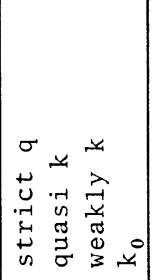 & 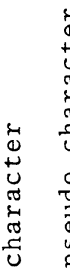 & \\
\hline 1 & $\mathrm{P}$ & $\begin{array}{l}\text { HHHHH- } \\
\mathrm{HHHHH}- \\
\mathrm{HHHHH}- \\
\mathrm{HHHHH}-\end{array}$ & $\begin{array}{l}\mathrm{HH} \\
\mathrm{HH} \\
\mathrm{HH} \\
\mathrm{HH}\end{array}$ & $\begin{array}{l}\mathrm{HHHH} \\
\mathrm{HHHH} \\
\mathrm{HHHH} \\
\mathrm{HHHH}\end{array}$ & $\begin{array}{l}\mathrm{HHHHH} \\
-\mathrm{HHHH} \\
\mathrm{HHHHH} \\
-\mathrm{HHHH}\end{array}$ & $\begin{array}{l}\mathrm{HHHH} \\
\mathrm{HHHH} \\
\mathrm{HHHH} \\
-\mathrm{HHH}\end{array}$ & $\begin{array}{ll}c & \\
c & \\
x_{1} & N \\
c & N\end{array}$ & \\
\hline $\begin{array}{l}5 \\
6 \\
7\end{array}$ & $\mathrm{~K}$ & $\begin{array}{l}\mathrm{HHHHH}- \\
\mathrm{HHHHH}- \\
--\mathrm{HHH}- \\
-\mathrm{HHHH}-\end{array}$ & $\begin{array}{l}\mathrm{HH} \\
\mathrm{HH} \\
-\mathrm{H} \\
\mathrm{HH}\end{array}$ & $\begin{array}{l}\mathrm{HHHH} \\
\mathrm{HHHH} \\
-\mathrm{HHH} \\
\mathrm{HHHH}\end{array}$ & $\begin{array}{l}-\mathrm{HHHH} \\
-\mathrm{HHHH} \\
---\mathrm{HH} \\
\mathrm{HHHHH}\end{array}$ & $\begin{array}{l}-\mathrm{HHH} \\
-\mathrm{HHH} \\
-\mathrm{HHH} \\
\mathrm{HHHH}\end{array}$ & $\begin{array}{l}\mathrm{c} \\
\mathrm{c} \\
{ }^{\mathrm{c}} \\
\text { meas }\end{array}$ & $\begin{array}{l}x_{0} \\
x_{0}\end{array}$ \\
\hline $\begin{array}{r}9 \\
10 \\
11 \\
12\end{array}$ & $\begin{array}{r}\mathrm{RC} \\
\mathrm{P}\end{array}$ & $\begin{array}{l}--\mathrm{HHH}- \\
--\mathrm{HHH}- \\
--\mathrm{HHH}- \\
--\mathrm{HHH}-\end{array}$ & \begin{tabular}{l|}
$-\mathrm{H}$ \\
$-\mathrm{H}$ \\
$-\mathrm{H}$ \\
$-\mathrm{H}$
\end{tabular} & $\begin{array}{l}-\mathrm{HHH} \\
-\mathrm{HHH} \\
-\mathrm{HHH} \\
-\mathrm{HHH}\end{array}$ & $\begin{array}{l}---\mathrm{HH} \\
---\mathrm{HH} \\
---\mathrm{HH} \\
---\mathrm{HH}\end{array}$ & $\begin{array}{l}-\mathrm{HHH} \\
-\mathrm{HHH} \\
-\mathrm{HHH} \\
-\mathrm{HHH}\end{array}$ & $\begin{array}{ll}\mathrm{c} & \mathrm{x} \\
\mathrm{c} & \mathrm{x} \\
\mathrm{c} & \mathrm{x} \\
\mathrm{c} & \mathrm{x}\end{array}$ & \\
\hline $\begin{array}{l}13 \\
14 \\
15 \\
16\end{array}$ & $\begin{array}{c}3.5 \\
P \\
P \\
P\end{array}$ & $\begin{array}{l}-\mathrm{HHH}- \\
--\mathrm{HHH}- \\
--\mathrm{HHH}- \\
--\mathrm{HHH}-\end{array}$ & \begin{tabular}{l|}
$-\mathrm{H}$ \\
$-\mathrm{H}$ \\
$-\mathrm{H}$ \\
$-\mathrm{H}$
\end{tabular} & $\begin{array}{l}-\mathrm{HHH} \\
-\mathrm{HHH} \\
-\mathrm{HHH} \\
-\mathrm{HHH}\end{array}$ & $\begin{array}{l}---\mathrm{HH} \\
---\mathrm{HH} \\
---\mathrm{HH} \\
---\mathrm{HH}\end{array}$ & $\begin{array}{l}-\mathrm{HHH} \\
-\mathrm{HHH} \\
-\mathrm{HHH} \\
-\mathrm{HHH}\end{array}$ & $\begin{array}{l}\text { c } \\
\mathrm{c} \\
\mathrm{c} \\
\mathrm{c}\end{array}$ & $\begin{array}{l}x_{0} \\
x_{0} \\
\kappa_{0}\end{array}$ \\
\hline $\begin{array}{l}17 \\
18 \\
19 \\
20\end{array}$ & $\begin{array}{c}\mathrm{P} \\
\mathrm{RC}\end{array}$ & $\begin{array}{r}--\mathrm{HHH}_{-} \\
--\mathrm{HHH}- \\
\mathrm{HHH}- \\
--\mathrm{HHH}-\end{array}$ & $\begin{array}{r}-H \\
-H \\
H \\
-H\end{array}$ & $\begin{array}{r}-\mathrm{HHH} \\
-\mathrm{HHH} \\
\mathrm{HHH} \\
-\mathrm{HHH}\end{array}$ & $\begin{array}{r}---H H \\
---H H \\
H H \\
--H H\end{array}$ & $\begin{array}{r}-\mathrm{HHH} \\
-\mathrm{HHH} \\
\mathrm{HHH} \\
-\mathrm{HHH}\end{array}$ & $\begin{array}{l}\mathrm{c} \\
\mathrm{c} \\
2^{c}\end{array}$ & \\
\hline \begin{tabular}{l|}
21 \\
22 \\
23 \\
24
\end{tabular} & \begin{tabular}{|l|}
$\mathrm{K}$ \\
$\mathrm{P}$ \\
$\mathrm{P}$
\end{tabular} & $\begin{array}{l}---+H- \\
---+H+ \\
---+H+ \\
---+H+\end{array}$ & $\begin{array}{l}-- \\
-- \\
-- \\
--\end{array}$ & $\begin{array}{l}+++- \\
---- \\
---- \\
---\end{array}$ & $\begin{array}{l}++++t \\
----t \\
----+ \\
----+\end{array}$ & $\begin{array}{l}t+t+ \\
-t+t \\
-t+t \\
-t+t\end{array}$ & $\begin{array}{ll}\mathrm{c} & \\
\mathrm{c} & \mathrm{b} \\
\mathrm{c} & : \\
\mathrm{c} & :\end{array}$ & \\
\hline
\end{tabular}




\begin{tabular}{|c|c|c|c|c|c|c|c|}
\hline & & 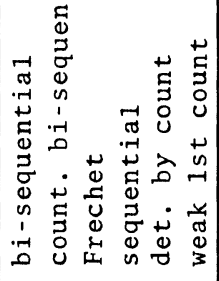 & 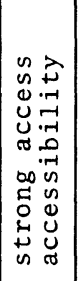 & 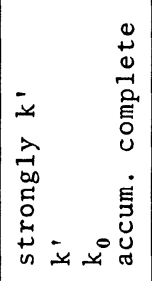 & 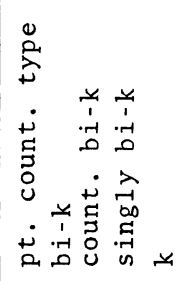 & 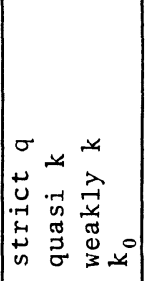 & 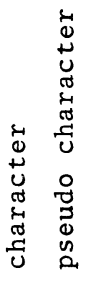 \\
\hline 25 & $\mathrm{RC}$ & ---+ & -- & ---- & --- & & $\kappa_{0}$ \\
\hline 26 & 2 & ---+ & -- & $--\cdots$ & --- & & $\kappa_{0}$ \\
\hline 27 & $\mathrm{P}$ & ---+ & -- & ---- & ---+ & & $\kappa_{0}$ \\
\hline 28 & 2 & ---+1 & -- & --- & ----+ & & $\kappa_{0}$ \\
\hline 29 & 2 & ---+1 & -- & --- & ----+ & & $\kappa_{0}$ \\
\hline 30 & $\mathrm{RC}$ & ---+1 & -- & ---- & ----+ & & $\kappa_{0}$ \\
\hline 31 & 3.5 & $---+\mathrm{H}+$ & -- & --- & ---+ & & $K^{\prime} 0$ \\
\hline 32 & $\mathrm{RC}$ & $---+H$ & -- & --- & $---\cdots+$ & $-t$ & $\kappa_{0}$ \\
\hline 33 & 2 & ---+1 & -- & ---- & ---++ & & $\dot{x}_{0}$ \\
\hline 34 & 2 & ---+ & -- & ---- & $-\cdots-+$ & & $\kappa_{0}$ \\
\hline 35 & $\mathrm{P}$ & ---+ & -- & --- & $-\cdots+-+$ & & $\kappa_{0}$ \\
\hline 36 & $\mathrm{P}$ & ---+1 & -- & $--\quad-$ & ---+ & & \\
\hline 37 & 2 & -1 & & & $-\quad-1$ & $t-$ & $\boldsymbol{x}_{0}$ \\
\hline 38 & $\mathrm{RC}$ & --- & $\mathrm{HH}$ & --- & $----\cdots$ & --- & $\kappa_{0}$ \\
\hline 39 & $\mathrm{RC}$ & ---- & $\mathrm{HH}$ & ---- & ---- & $-\cdots$ & $\kappa_{0}$ \\
\hline 40 & $\mathrm{RC}$ & $---\mathrm{H}-$ & -- & --- & ---- & ---- & $\kappa_{0}$ \\
\hline 41 & $\mathrm{RC}$ & ----1 & $\mathrm{HH}$ & $--\cdots$ & ---- & --- & $\kappa_{0}$ \\
\hline 42 & $\mathrm{RC}$ & $----\mathrm{H}-$ & $-\mathrm{H}$ & --- & $--\cdots$ & $-\cdots$ & $\kappa_{0}$ \\
\hline 43 & 2 & ---1 & $\mathrm{H}$ & ---- & $-\cdots-\cdots$ & - & c $\kappa_{0}$ \\
\hline 44 & 2 & $----\mathrm{H}-$ & & --- & $--\cdots-$ & ---- & $\kappa_{0}$ \\
\hline 45 & 2 & $----\mathrm{H}-$ & & - & & $-1+t$ & \\
\hline 46 & $\mathrm{RC}$ & -- & & & --- & - & $\kappa_{0}$ \\
\hline 47 & $\mathrm{P}$ & $----\mathrm{H}-$ & & --- & $---\cdots$ & - & \\
\hline 48 & 2 & $---\cdots$ & $\mathrm{HH}$ & $--\mathrm{HH}$ & $-\cdots-\cdots$ & $--1-1$ & $\kappa_{0}$ \\
\hline
\end{tabular}




\begin{tabular}{|c|c|c|c|c|c|c|c|}
\hline & & 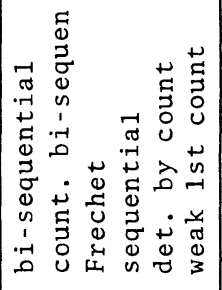 & $\begin{array}{ll}0 & 7 \\
0 & 0 \\
0 & -7 \\
0 & -1 \\
0 & -1 \\
0 & 0 \\
00 & -1 \\
00 & 0 \\
0 & 0 \\
0 & 0 \\
0 & 0 \\
0 & 0 \\
\omega & 0\end{array}$ & 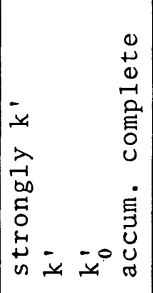 & 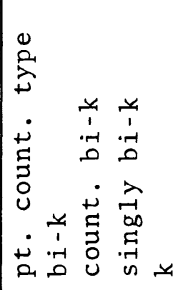 & 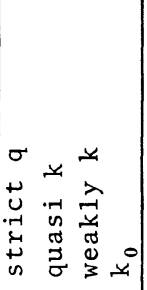 & 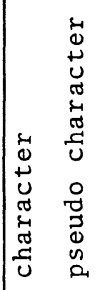 \\
\hline 49 & & $--\cdots-\cdots$ & $-\mathrm{H}$ & $--\mathrm{HH}$ & -- & & $N_{1} \kappa_{1}$ \\
\hline 50 & $\mathrm{P}$ & ----- & $-\mathrm{H}$ & $--\mathrm{HH}$ & -- & & c c \\
\hline 51 & K & ----- & -- & $+t+-$ & ++ & & $\kappa_{1} \kappa_{1}$ \\
\hline 52 & $\mathrm{~K}$ & $-\cdots-\cdots$ & -- & $+t$ & +1 & $4-1$ & $N_{1} \kappa_{1}$ \\
\hline 53 & $\mathrm{~K}$ & $-\cdots-\cdots$ & -- & $t+$ & +1 & & c c \\
\hline & K & $---\cdots$ & -- & & & & c \\
\hline 55 & K & $-\cdots-\cdots$ & -1 & & & & c \\
\hline 56 & K & $---\cdots$ & -1 & $+t$ & & & c \\
\hline 57 & $\mathrm{~K}$ & ----- & -- & $t+t-$ & ++1 & & $\begin{array}{|ll|} & c \\
\end{array}$ \\
\hline 58 & $\mathrm{~K}$ & $-\cdots-\cdots$ & -1 & & & & $N_{1} N_{1}$ \\
\hline 59 & K & & & $-1-1$ & & & \\
\hline 603 & 3.5 & ----- & -- & $+t+-$ & $+t+t+$ & & $N_{1} \kappa_{1}$ \\
\hline & 3.5 & & & $-1+1$ & $-1-1-1$ & & $x_{1} \kappa_{1}$ \\
\hline 623 & 3.5 & & & $-1-1$ & $-1-1-1$ & & $\kappa_{1} \kappa_{1}$ \\
\hline 633 & 3.5 & $--\cdots--$ & -- & $+t+-$ & $t+t+$ & & $x_{1} x_{1}$ \\
\hline 64 & $\mathrm{P}$ & ----- & -- & $-4+$ & ---++ & & $2^{\mathrm{N}_{1}} \mathrm{~N}_{1}$ \\
\hline 65 & 2 & $--\cdots--$ & -- & $--\mathrm{HH}$ & $----\cdots$ & $--\mathrm{H}$ & $\kappa_{0}$ \\
\hline & & $--\cdots--$ & -- & -- & $t+t+t$ & $-1-t-1$ & \\
\hline 673 & 3.5 & $--\infty \quad-$ & -- & -- & & & \\
\hline 683 & 3.5 & $---\cdots$ & & $--\cdots$ & ----- & $-1-1--$ & c \\
\hline 693 & 3.5 & $----\cdots$ & & -- & ----- & - & c \\
\hline & 4 & $--\cdots-$ & & -- & $-\cdots-\cdots$ & & \\
\hline 713 & 3.5 & ---- & & -- & ---- & -1 & \\
\hline 723 & 3.5 & ---- & & -- & $-\cdots-\cdots$ & - & \\
\hline
\end{tabular}




\section{BIBLIOGRAPHY}

1. O. T. Alas, Uniform continuity in paracompact spaces, in: General Topology and its Relations to Modern Analysis and Algebra III. Proceedings of the Third Prague Topological Symposium, 1971, Academic Press, New York, 1972, pp. 19-22.

2. P. S. Alexandroff and P. S. Urysohn, On compact topological spaces, Akad. Nauk SSSR, Trudi Mat. Inst. Steklova, vol. 31, 1950 (Kraus Reprint LTD., Vaduz, 1963). (This is a revision by Alexandroff in Russian of Memoire sur les espaces topologiques compacts, Verh. Akad. Wetensch, Amsterdam 14 (1929), 196.)

3. K. Alster, Remarks on compact-covering mappings, Bull. Acad. Polon. 19 (1971), 141-148.

4. B. A. Anderson, A class of topologies with $T_{1}$-complements, Fund. Math. 69 (1970), 267-277.

5. D. R. Anderson, On connected irresolvable Hausdorff spaces, Proc. Amer. Math. Soc. 16 (1965), 463-466.

6. F. W. Anderson, A lattice characterization of completely regular $G_{\delta^{-}}$ spaces, Proc. Amer. Math. Soc. 6 (1955), 757-765. 209.

7. P. Antosik, On a topology of convergence, Colloq. Math. 21 (1970), 205-

8. R. Arens, A topology for spaces of transformations, Ann. of Math. 47 (1946), 480-495.

9. - Note on convergence in topology, Math. Mag. 23 (1950), 229-234.

10. A. Arhangel'skii, Concerning the weight of topological spaces, General Topology and its Relations to Modern Analysis and Algebra. Proceedings of the Symposium held in Prague in 1961, Academic Press, New York, 1962, pp. 72-74.

11. - Bicompact sets and the topology of spaces, Soviet Math. Dokl. 4 (1963), 561-564.

12. - On a class of spaces containing all metric and all locally bicompact spaces, Soviet Math. Dokl. 4 (1963), 1051-1055.

13. - Some types of factor mappings, and the relations between classes of topological spaces, Soviet Math. Dokl. 4 (1963), 1726-1729.

14. —, Factor mappings of metric spaces, Soviet Math. Dokl. 5 (1964), $368-371$.

15. - Bicompact sets and the topology of spaces, Trans. Moscow Math. Soc. 13 (1965), 1-62.

16. - On closed mappings, bicompact spaces, and a problem of $P$. Aleksandrov, Pacific J. Math. 18 (1966), 201-208.

17. — On embeddings of $T_{1}$-spaces in bicompact $T_{1}$-spaces of the same weight, Bull. Acad. Polon. 14 (1966), 361-366.

18. — Mappings and spaces, Russian Math. Surveys 21 (1966), 115-162.

19. - On some results concerning $k$-spaces, in: General Topology and its Relations to Modern Analysis and Algebra. Proceedings of the Second Prague Topological Symposium, 1966, Academic Press, New York, 1967, p. 42.

20. _ - A theorem on the metrizability of the inverse image of a metric space under an open-closed finite-to-one mapping. Example and unsolved problems, Soviet Math. Dokl. 7 ( (1966), 1258-1262.

21. - Every extremally disconnected bicompactum of weight $c$ is inhomogeneous, Soviet Math. Dokl. 8 (1967), 897-900. 
22. $1217-1220$.

23. — A characterization of very $k$-spaces, Czech. Math. J. 18 (1968), 392-395.

24. - - Closed mappings, bicompact sets and a problem of P. S. Aleksandrov, Translations Amer. Math. Soc. 78 (1968), 41-66.

25. — Mappings related to topological groups, Soviet Math. Dokl. 9 (1968), 1011-1015.

26. — On the cardinality of bicompacta satisfying the first axiom of countability, Soviet Math. Dokl. 10 (1969), 951-955.

27. - On a class of spaces containing all metric spaces and all locally bicompact spaces, Translations Amer. Math. Soc. 92 (1970), 1-39.

28. — - On bicompacta hereditarily satisfying Souslin's condition. Tightness and free sequences, Soviet Math. Dokl. 12 (1971), 1253-1257.

29. —_ Proposed problems, General Topology and Appl. 1 (1971), ix.

30. — - On cardinal invariants, General Topology and its Relations to Modern Analysis and Algebra III. Proceedings of the Third Prague Topological Symposium, 1971, Academic Press, New York, 1972, pp. 37-46.

31. - The frequency spectrum of a topological space and the classification of spaces, Soviet Math. Dokl. 13 (1972), 1185-1189.

32. —_, There is no "naive" example of a nonseparable sequential bicompactum with the Suslin property, Soviet Math. Dokl. 13 (1972), 473-476.

33. — - On hereditary properties, General Topology and Appl. 3 (1973), 39-46.

34. A. Arhangel'skii and S. P. Franklin, Ordinal invariants for topological spaces, Mich. Math. J. 15 (1968), 313-320.

35. A. Arhangel'skii and V. I. Ponomarev, On dyadic bicompacta, Soviet Math. Dokl. 9 (1968), 1220-1224.

36. S. P. Arya, A note on nearly paracompact spaces, Mat. Vesnik 8 (23) (1971), 113-115.

37. C. E. Aull, A certain class of topological spaces, Prace Mat. 11 (1967), 49-53.

38. - Classification of topological spaces, Bull. Acad. Polon. 15 (1967), 773-778.

39. _ - Sequences in topological spaces, Prace Mat. 11 (1968), 329-336.

40. - Sequences and Stone-Cech compactifications, Not. Amer. Math. Soc. 16 (1969), 851 \#69T-G113.

41. — Properties of side points of sequences, General Topology and Appl. l (1971), 201-208.

42. — Some base axioms for topology involving enumerability, General Topology and its Relations to Modern Analysis and Algebra. Proceedings of the Kanpur Topological Conference, 1968, Academia, Prague, 1971, pp. 55-61.

43. — A separation axiom of F. Riesz, preprint.

44. —, A note on $k$ spaces, Preliminary report, Not. Amer. Math. Soc. 20 (1973), A-533 \#706-54-3.

45. P. C. Baayen and M. A. Maurice, Johannes de Groot, 1914-1972, General Topology and Appl. 3 (1973), 1-32.

46. M. Bae, $P_{1}$-mapping and property $K$, Kyungpook Math. J. 3 (1960), 39-41.

47. R. W. Bagley and D. D. Weddington, Products of $k^{\prime}$-spaces, Proc. Amer. Math Soc. 22 (1969), 392-394.

48. R. W. Bagley and J. S. Yang, On k-spaces and function spaces, Proc. Amer. 
Math. Soc. 17 (1966), 703-705.

49. J. W. Baker, Ordinal subspaces of topological spaces, General Topology and Appl. 3 (1973), 85-91.

50. K. A. Baker, Hypotopological spaces and their embeddings in lattices with Birkhoff interval topology, Pacific J. Math. 28 (1969), 275-288.

51. V. K. Balachandran, A mapping theorem for metric spaces, Duke Math. J. 22 (1955), 461-464.

52. S. Baron, The coreflective subcategory of sequential spaces, Canad. Math. Bull. 11 (1968), 603-604.

53. S. Baron and S. Leader, Sequential topologies, Amer. Math. Monthly 73 (1966), 677-678 \#5299.

54. A. I. Baskirov, Topological spaces determined by their subspaces, Soviet Math. Dokl. 13 (1972), 1639-1643.

55. A. R. Bednarek and J. Mikusinski, Convergence and topology, Bull. Acad. Polon. 17 (1969), 437-442.

56. D. W. Behrens, Adjunction spaces and $k$-spaces, Not. Amer. Math. Soc. 20 (1973), A-176 \#701-54-27.

57. K. Bierstedt, Gewichtete Raume stetiger vektorwertiger Funktionen und das injektive Tensorprodukt. I. J. Reine Angew Math. 259 (1973), 186-210. print.

58. H. J. Biesterfeldt, The first axiom of cardinality, Univ. of Oklahoma pre-

59. L. J. Billera, Topologies for $2^{X}$; set-valued functions and their graphs, Trans. Amer. Math. Soc. 155 (1971), 137-147.

60. R. H. Bing, Metrization of topological spaces, Canad. J. Math. 3 (1951), 175-186.

61. G. Birkhoff, On the combination of topologies, Fund. Math. 26 (1936), $156-166$.

62. T. K. Boehme, Linear s-spaces, Symposium on Convergence Structures, Univ. of Oklahoma, March 1965, pp. 1-7.

63. J. R. Boone, A metrization theorem for developable spaces, Fund. Math. 73 (1971), 79-83.

64. - Some characterizations of paracompactness in k-spaces, Fund. Math. 72 (1971), 145-153.

65. —- Examples relating to mesocompact and sequentially mesocompact spaces, Fund. Math. 77 (1972), 91-93.

66. - On k-quotient mappings, Preprint.

67. J. R. Boone and F. Siwiec, Sequentially quotient mappings, Not. Amer. Math. Soc. 20 (1973), A-598 \#73T-G139.

68. P. I. Booth, The section problem and the lifting problem, Math. Zeit. 121 (1971), 273-287.

69. C. J. R. Borges, On stratifiable spaces, Pacific J. Math. 17 (1966), 1-16.

70. - Metrizability of adjunction spaces, Proc. Amer. Math. Soc. 24 (1970), 446-451.

71. - A survey of $M_{i}$-spaces: Open questions and partial results, General Topology and Appl. 1 (1971), 79-84.

72. N. Bourbaki, Topologie Générale, Ch. 9, second edition, Paris, 1958.

73. R. C. Briggs, III, Preparacompactness and $\mathrm{x}$-preparacompactness in $q$ spaces, Colloq. Math. 27 (1973), 227-235.

74. R. Brown, Ten topologies for $X \times Y$, Quart. J. Math. 14 (1963), 303-319.

75. — Function spaces and product topologies, Quart. J. Math. 15 (1964), 238-250. 
76. H. Buchwalter, Sur une famille particulière de bornés d'un espace topologique complètement régulier, C. R. Acad. Sc. Paris 273 (1971), 228-231.

77. D. Burke, On p-spaces and $w$-spaces, Pacific J. Math. 35 (1970), 285-296.

78. D. Burke, R. Engelking, and D. Lutzer, Hereditarily closure-preserving collections and metrization, Preprint.

79. D. Burke and R. A. Stoltenberg, Some properties of $\pi$-images of metric spaces, unpublished.

80. D. E. Cameron, Maximal pseudocompactness, in: Topology Conference at Emory Univ., 1970, pp. 26-31.

81. - Maximal and minimal topologies, Trans. Amer. Math. Soc. 160 (1971), 229-248.

82. E. Cech, rev. ed. by Z. Frolik and M. Katetov, Topological Spaces, John Wiley, New York, 1966.

83. J. G. Ceder, Some generalizations of metric spaces, Pacific J. Math. 11 (1961), 105-125.

84. - - On maximally resolvable spaces, Fund. Math. 55 (1964), 87-93.

85. — - On maximally resolvable spaces, in: Topology Conference, Arizona State University, 1967, pp. 55-57.

86. J. G. Ceder and T. Pearson, On products of maximally resolvable spaces, Pacific J. Math. 22 (1967), 31-45.

87. J. Chaber, Remarks on open-closed mappings, Fund. Math. 74 (1972), 197-208.

88. T. Chiba, On q-spaces, Proc. Japan Acad. 45 (1969), 453-456.

89. E. W. Chittenden, On general topology and the relation of the properties of the class of all continuous functions to the properties of space, Trans. Amer. Math. Soc. 31 (1929), 290-321.

90. M. Coban, Metrizability behavior in the case of factor s-mappings, Soviet Math. Dokl. 7 (1966), 141-144.

91. — On perfect mappings and spaces of countable type, Vestnik Moskov Univ. (1967), \#6, 87-93 (in Russian).

92. — On spaces of bicompact subsets, Moscow Univ. Math. Bull. 24 (1969), \#2, 44-46. 1260.

93. — - On the theory of p-spaces, Soviet Math. Dokl. 11 (1970), 1257-

94. D. E. Cohen, Spaces with weak topology, Quart. J. Math. (2) 5 (1954), 77-80.

95. _ _ Products and carrier theory, Proc. London Math. Soc. (3) 7 (1957), 219-248.

96. H. S. Collins, Completeness, full completeness, and $k$-spaces, Proc. Amer. Math. Soc. 6 (1955), 832-835.

97. W. W. Comfort, Locally compact realcompactifications, in: General Topology and its Relations to Modern Analysis and Algebra. Proceedings of the Second Prague Topological Symposium, 1966, Academic Press, New York, 1967, pp. 95-100.

98. — On the Hewitt realcompactification of a product space, Trans. Amer. Math. Soc. 131 (1968), 107-118.

99. — A survey of cardinal invariants, General Topology and Appl. 1 (1971), 163-199.

100. W. W. Comfort and S. Negrepontis, Homeomorphs of three subspaces of $\beta N-N$, Math. Zeit. 107 (1968), 53-58. 
101. J. B. Conway, The inadequacy of sequences, Amer. Math. Monthly 76 (1969), 68-69.

102. W. F. Davison, Mosaics of compact metric spaces, Trans. Amer. Math. Soc. 91 (1959), 525-546.

103. E. J. Dubuc and H. Porta, Convenient categories of topological algebras, Bull. Amer. Math. Soc. 77 (1971), 975-979.

104. E. Duda, A locally compact metric space is almost invariant under a closed mapping, Proc. Amer. Math. Soc. 16 (1965), 473-475.

105. — Reflective compact mappings, Proc Amer. Math. Soc. 17 (1966), 688-693.

106. R. M. Dudley, On sequential convergence, Trans. Amer. Math. Soc. 112 (1964), 483-507.

107. B. Dushnik, Concerning Fréchet limit-spaces, Fund. Math. 23 (1934), 162-165.

108. B. A. Efimov, Dyadic bicompacta, Trans. Moscow Math. Soc. (1965), 229267.

109. - On the power of Hausdorff spaces, Soviet Math. Dokl. 6 (1965), 1315-1318.

110. - On imbedding of Stone-Cech compactifications of discrete spaces in bicompacta, Soviet Math. Dokl. 10 (1969), 1391-1394.

111. - Extremally disconnected compact spaces and absolutes, Trans. Moscow Math. Soc. 23 (1970), 243-285.

112. B. A. Efimov and V. M. Kuznecov, On topological types of dyadic spaces, Soviet Math. Dokl. 11 (1970), 1403-1407.

113. A. G. El'kin, On irresolvable spaces and ultrafilters that are topologically dense in themselves, Soviet Math. Dokl. 10 (1969), 1424-1427.

114. - On the maximal resolvability of products of topological spaces, Soviet Math. Dokl. 10 (1969), 659-662.

115. V. V. Filippov, On factor s-mappings, Soviet Math. Dokl. 9 (1968), 10551057.

116. - Quotient spaces and multiplicity of a base, Math. USSR-Sbornik 9 (1969), 487-496.

117. I. Fleischer and S. P. Franklin, On compactness and projections, in: Contributions to Extension Theory of Topological Structures, Berlin, 1969, pp. 77-79.

118. S. P. Franklin, Spaces in which sequences suffice, Fund. Math. 57 (1965), 107-115.

119. - On unique sequential limits, Nieuvw Archief voor Wiskunde (3) 14 (1966), 12-14.

120. - Spaces in which sequences suffice. II, Fund. Math. 61 (1967), $51-56$.

121. - Natural covers, Comp. Math. 21 (1969), 253-261.

122. - On two questions of Moore and Mrowka, Proc. Amer. Math. Soc. 21 (1969), 597-599.

123. — Topics in categorical topology, Lecture notes, 1970.

124. —, A homogeneous Hausdorff $E_{0}$-space which is not $E_{1}$, in: General Topology and its Relations to Modern Analysis and Algebra III, Proceedings of the Kanpur Topology Conference, 1968, Prague, 1971.

125. S. P. Franklin and J. K. Kohli, On open extensions of maps, Canad. J. Math. 22 (1970), 691-696.

126. S. P. Franklin and M. Rajagopalan, Some examples in topology, Trans. Amer. Math. Soc. 155 (1971), 305-313. 
127. S. P. Franklin and R. H. Sorgenfrey, Closed and image-closed relations, Pacific J. Math. 19 (1966), 433-439.

128. R. Fric, A note on Fréchet spaces, Comm. Math. Univ. Carolinae 13 (1972), 411-418.

129. A. Frölicher, Sur l'anneau des fonctions continues d'un espace à génération compacte, C. R. Acad. Sc. Paris 275 (1972), 25-27.

130. - Kompakt erzengte Räume und Limesräume, Math. Zeit. 129 (1972), 57-63.

131. A. Frölicher and M. Roulin, Topologies faibles et topologies a génération compacte, L'Enseignement Math. 18 (1972), 205-207.

132. Z. Frolik, Topologically complete spaces, Comment. Math. Univ. Carolinae 1 (1960), \#3, 3-15.

133. R. V. Fuller, Relations among continuous and various non-continuous functions, Pacific J. Math. 25 (1968), 495-509.

134. P. Gabriel and M. Zisman, Calculus of Fractions and Homotopy Theory, Springer, New York, 1967.

135. D. Gale, Compact sets of functions and function rings, Proc. Amer. Math. Soc. 1 (1950), 303-308.

136. L. Gillman and M. Jerison, Rings of Continuous Functions, Van Nostrand, Princeton, 1960.

137. A. Goetz, A notion of uniformity for L-spaces of Frechet, in: General Topology and its Relations to Modern Analysis and Algebra I. Proceedings of the Prague Topology Conference, 1961, pp. 177-178.

138. — - Proximity-like structures in convergence spaces, in: Topology Conference, Arizona State Univ., 1967, pp. 104-137.

139. G. K. Goss, Weight and pseudo-weight of minimal Hausdorff spaces, Not. Amer. Math. Soc. 17 (1970), 846 \#70T-G146.

140. J. de Groot, An isomorphism principle in general topology, Bull. Amer. Math. Soc. 73 (1967), 465-467.

141. G. Gruenhage, A separable continuously normal space is metrizable, Not. Amer. Math. Soc. 20 (1973), A-452 \#73T-G75.

142. S. L. Gulden, On m-sequential spaces, Not. Amer. Math. Soc. 16 (1969), 293 \#663-706.

143. J. A. Guthrie, A characterization of $\boldsymbol{\kappa}_{0}$-spaces, General Topology and Appl. 1 (1971), 105-110.

144. - Ascoli theorems and the pseudocharacter of mapping spaces. Preliminary report, Not. Amer. Math. Soc. 20 (1973), A-457 \#73T-G92.

145. _ - Mapping spaces and cs-networks, Pacific J. Math. 47 (1973), 465471.

146. J. A. Guthrie and M. Henry, Sequential spaces and quasiperfect mappings, Preprint.

147. J. A. Guthrie and H. E. Stone, Expansions of mappings, Preprint.

148. A. Hajnal and I. Juhasz, Discrete subspaces of topological spaces, Indag. Math. 29 (1967) = Proc. Nederl. Akad., Series A 70 (1967), 343-356.

149. - Some results in set-theoretic topology, Soviet Math. Dokl. 8 (1967), 141-143.

150. E. Halfar, Conditions implying continuity of functions, Proc. Amer. Math. Soc. 11 (1960), 688-691.

151. S. Hanai, On closed mappings, Proc. Japan Acad. 30 (1954), 285-288.

152. - Inverse images of closed mappings. I, Proc. Japan Acad. 37 (1961), 298-301. 
153. — - On open mappings. II, Proc. Japan Acad. 37 (1961), 233-238.

154. P. W. Harley, III, Metrization of closed images of metric spaces, Not. Amer. Math. Soc. 19 (1972), A-607 \#72T-G125.

155. - Products of Fréchet spaces, Not. Amer. Math. Soc. 19 (1972), A-378 \#692-G20.

156. - Closed images of metric spaces, Not. Amer. Math. Soc. 20 (1973), A-29 \#73T-G21.

157. D. Harris, Transfinite metrics, sequences. and topological properties, Fund. Math. 73 (1971), 137-142.

158. B. V. Hearsey, Regularity in convergence spaces, Port. Math. 30 (1971), $201-213$

159. B. V. Hearsey and D. C. Kent, Convergence structures, Port. Math. 31 (1972), 105-118.

160. R. W. Heath, On certain first countable spaces, in: Topology Seminar, Wisconsin, 1965, ed. by Bing and Bean, pp. 103-113.

161. —, On p-spaces, q-spaces, r-spaces and " $s$ " spaces, in: Proc. Auburn Conf., March 1969, pp. 123-134.

162. - An easier proof that a certain countable space is not stratifiable, in: Proc. Washington State Univ. Conf., March 1970, pp. 56-59.

163. - On a non-stratifiable countable space, in: Topology Conf., Emory Univ., 1970, pp. 119-122.

164. L. J. Heider, A note concerning completely regular $G_{\delta}$-spaces, Proc. Amer. Math. Soc. 8 (1957), 1060-1066.

165. M. Henriksen and J. R. Isbell, Some properties of compactifications, Duke Math. J. 25 (1958), 83-105.

166. D. M. Henry, On the images of a stratifiable space under certain open and pseudo-open mappings, Ph.D. Thesis, Texas Christian Univ., Fort Worth, 1970.

167. —- Conditions for countable base in spaces of countable and pointcountable type, Pacific J. Math. 36 (1971), 181-183.

168. H. Herrlich, Quotienten geordneter Räume und Folgenkonvergenz, Fund. Math. 61 (1967), 79-81.

169. - Limit-operators and topological coreflections, Trans. Amer. Math. Soc. 146 (1969), 203-210.

170. - Categorical topology, General Topology and Appl. 1 (1971), 1-15.

171. H. Herrlich and J. van der Slot, Properties which are closely related to compactness, Indag. Math. $29(1967)$ = Proc. Nederl. Akad., Series A 70 (1967), 524-529.

172. H. Herrlich and G. E. Strecker, Coreflective subcategories in general topology, Fund. Math. 73 (1972), 199-218.

173. R. E. Hodel, Spaces defined by sequences of open covers which guarantee that certain sequences have cluster points, Duke Math. J. 39 (1972), 253-263.

174. V. D. House, Countable products of generalized countably compact spaces. Preliminary report, Not. Amer. Math. Soc. 19 (1972), A-541 \#72T-G97.

175. N. Howes and R. Chandler, Accumulation complete and sequential spaces, Not. Amer. Math. Soc. 16 (1969), 850 \#69T-G109.

176. M. Hukuhara and Y. Sibuya, Sur l'endomorphisme complètement continu, Proc. Japan Acad. 31 (1955), 595-599.

177. A. Hulanicki, On the power of compact spaces, Colloq. Math. 7 (1960), 199-200. 
178. M. Husek, Products of quotients and of $k^{\prime}$-spaces, Comm. Math. Univ. Carolinae 12 (1971), 61-68.

179. — Products as reflections, Comm. Math. Univ. Carolinae 13 (1972), 783-800.

180. - Realcompactness of function spaces and $v(P \times Q)$, General Topology and Appl. 2 (1972), 165-179.

181. T. Ishii, $w M$-spaces and closed maps, Proc. Japan Acad. 46 (1970), 16-21.

182. T. Isiwata, Normality and perfect mappings, Proc. Japan Acad. 39 (1963), 95-97.

183. - The product of M-spaces need not be an M-space, Proc. Japan Acad. 45 (1969), 154-156.

184. F. B. Jones, On the first countability axiom for locally compact Hausdorff spaces, Colloq. Math. 7 (1959), 33-34.

185. I. Juhasz, On the character of points in $\beta N_{m}$, in: Proc. First International Symposium on Extension Theory, Berlin, 1967, pp. 139-140.

186. I. Juhasz, in collaboration with A. Verbeek and N. S. Kroonenberg, Cardinal Functions in Topology, Mathematical Centre Tract \#34, Amsterdam, 1971.

187. V. Kannan, Coreflexive subcategories in topology, Technical report, Madurai University, 1970.

188. — - Solutions to ten problems in topology, Preprint.

189. - On three questions of Arhangel'skii and Franklin, Not. Amer. Math. Soc. 17 (1970), 839-840 \#70T-G123.

190. —, E-Fréchet spaces, Not. Amer. Math. Soc. 18 (1971), 262 \#71T-G7.

191. —, Open problems, Preprint.

192. — An extension that nowhere has the Fréchet property, Michigan Math. J. 20 (1973), 225-234.

193. V. Kannan and M. Rajagopalan, On rigidity and groups of homeomorphisms, in: General Topology and its Relations to Modern Analysis and Algebra. Proceedings of the Third Prague Topological Symposium, 1971, Academic Press, New York, 1972, pp. 231-234.

194. S. Kasahara, Boundedness of semicontinuous finite real functions, Proc. Japan Acad. 33 (1957), 183-186.

195. M. Katetov, Remarks on characters and pseudocharacters, Comm. Math. Univ. Carolinae 1 (1960), \#1, 20-25.

196. — Convergence structures, in: General Topology and its Relations to Modern Analysis and Algebra. Proceedings of the Second Prague Topological Symposium, 1966, Academic Press, New York, 1967, pp. 207-216.

197. J. Keesling, On the equivalence of normality and compactness in hyperspaces, Pacific J. Math. 33 (1970), 657-667.

198. - Normality and compactness are equivalent in hyperspaces, Bull. Amer. Math. Soc. 76 (1970), 618-619.

199. J. L. Kelley, General Topology, Van Nostrand, Princeton, 1955.

200. J. L. Kelley and I. Namioka, Linear Topological Spaces, Van Nostrand, Princeton, 1963, pp. 76-81.

201. D. C. Kent, The interval topology and order convergence as dual convergence structures, Amer. Math. Monthly 74 (1967), 426-427, 1231.

202. - Convergence quotient maps, Fund. Math. 65 (1969), 197-205.

203. —, Decisive convergence structures, Math. Ann. 184 (1970), 215-223.

204. —, Spaces in which well ordered nets suffice, in: Proc. Washington State Univ. Conf., March 1970, pp. 87-101.

205. — - Decisive convergence spaces, Fréchet spaces and sequential 
spaces, Rocky Mountain J. Math. 1 (1971), 367-374.

206. D. C. Kent and G. D. Richardson, Minimal convergence spaces, Trans. Amer. Math. Soc. 160 (1971), 487-499.

207. — Locally compact convergence spaces, Not. Amer. Math. Soc. 19 (1972), A-656 \#696-54-14.

208. J. Kisyński, Convergence du type L, Colloq. Math. 7 (1960), 205-211.

209. J. A. Kofner, On a new class of spaces and some problems of symmetrizability theory, Soviet Math. Dokl. 10 (1969), 845-848.

210. — On pseudostratifiable spaces, Fund. Math. 70 (1971), 25-47 (in Russian).

211. A. P. Kombarov, Hereditarily disconnected spaces, Moscow Univ. Math. Bull. 26 (1971) \#4, 82-84.

212. - On the product of normal spaces, uniformities on $\Sigma$-products, Soviet Math. Dokl. 13 (1972), 1068-1071.

213. - On closed projections, Bull. Acad. Polon. 21 (1973), 519-521.

214. V. Koutnik, On convergence topologies, in: General Topology and its Relations to Modern Analysis and Algebra. Proceedings of the Second Prague Topological Symposium, 1966, Academic Press, New York, 1967, pp. 226-228.

215. T. R. Kramer, On product spaces, Not. Amer. Math. Soc. 18 (1971), 567 \#71 T-G69, and preprint.

216. B. Kripke, One more reason why sequences are not enough, Amer. Math. Monthly 74 (1967), 563-565.

217. K. Kuratowski, Topology, vol. I, Academic Press, New York, 1966.

218. R. E. Larson, On the lattice of topologies, Ph. D. Thesis, University of Colorado, 1970.

219. - A sublattice of the lattice of topologies, Not. Amer. Math. Soc. 17 (1970), 223 \#672-489.

220. — Complementary topological properties, Not. Amer. Math. Soc. 20 (1973), A-176 \#701-54-25.

221. R. E. Larson and S. J. Zimmerman, The lattice of topologies: a survey, Preprint.

222. N. Lasnev, Continuous decompositions and closed mappings of metric spaces, Soviet Math. Dokl. 6 (1965), 1504-1506.

223. — - Closed images of metric spaces, Soviet Math. Dokl. 7 (1966), 12191221.

224. L. D. Lavallee, Mosaic spaces, $P_{1}$-mappings, and property $K$, Boll. Un. Mat. Ital. (3) 19 (1964), 95-97.

225. J. L. Lawrence, Sequential limit spaces, Amer. Math. Monthly 60 (1953), 590.

226. Y. F. Lin and L. Soniat, A new characterization of Hausdorff $k$-spaces, Proc. Japan Acad. 44 (1968), 1031-1032.

227. W. F. Lindgren and P. Fletcher, Locally quasi-uniform spaces with countable bases, Preprint.

228. D. J. Lutzer, Semimetrizable and stratifiable spaces, General Topology and Appl. 1 (1971), 43-48.

229. R. Machuca, A coincidence theorem, Amer. Math. Monthly 74 (1967), 569.

230. J. E. Mack, Countable paracompactness and weak normality properties, Trans. Amer. Math. Soc. 148 (1970), 265-271.

231. K. D. Magill, Composable topological properties and semigroups of relations, Austral. J. Math. 11 (1970), 265-275. 
232. W. E. Malbon, Invariants for quasi-compact mappings, Ph.D. Thesis, Univ. of Virginia, 1955.

233. V. I. Malyhin, On countable spaces having no bicompactification of countable tightness, Soviet Math. Dokl. 13 (1972), 1407-1411.

234. V. J. Mancuso, Mesocompactness and related properties, Pacific J. Math. 33 (1970), 345-355.

235.—, Inverse-images and first countability, General Topology and Appl. 2 (1972), 29-44.

236. —_, Expansion and dimension, Not. Amer. Math. Soc. 21 (1974), A-213 \#711-54-18.

237. N. M. Marrach, Certain local properties of topological spaces, Diss. Abstr. 1969, 3831-B \#69-6600.

238. H. W. Martin, Product maps and countable paracompactness, Canad. J. Math. 24 (1972), 1187-1190.

239. R. H. Marty, m-adic spaces, Acta Math. Acad. Sci. Hungar. 22 (1971), 441-447.

240. — $\quad k$-R-spaces, Comp. Math. 25 (1972), 149-152.

241. S. Mazur, On continuous mappings on cartesian products, Fund. Math. 39 (1952), 229-238.

242. P. McDougle, A theorem on quasi-compact mappings, Proc. Amer. Math. Soc. 9 (1958), 474-477.

243. — , Mapping and space relations, Proc. Amer. Math. Soc. 10 (1959), 320-323.

244. E. R. McMillan, On continuity conditions for functions, Pacific J. Math. 32 (1970), 479-494.

245. P. R. Meyer, Sequential topologies, Trans. New York Acad. Sci. II 30 (1968), 645-648.

246. - Function spaces and the Aleksandrov-Urysohn conjecture, in: Proc. First International Symp. on Extension Theory, 1967, Berlin, 1969, pp. 149-151.

247. — Sequential properties of ordered topological spaces, Comp. Math. 21 (1969), 102-106.

248. - Function spaces and the Aleksandrov-Urysohn conjecture, Annali di Mat. 86 (1970), 25-30.

249. — - New cardinal invariants for topological spaces, in: General Topology and its Relations to Modern Analysis and Algebra. Proc. Kanpur Topology Conf., 1968, Academia, Prague, 1970, pp. 201-206.

250. - Sequential space methods in general topological spaces, Colloq. Math. 22 (1971), 223-228.

251. - On the cardinality of dense sets, Proc. Nederl. Akad. $75(1972)=$ Indag. Math. 34 (1972), 210-211.

252. - Intersections of metrizable topologies, Preprint.

253. — - On total orderings in topology, in: General Topology and its Relations to Modern Analysis and Algebra. Proceedings of the Third Prague Topological Symposium, 1971, Academic Press, New York, 1972, pp. 301-306.

254. - - When does countable compactness imply sequential compactness? Preprint.

255. — L Lattice operations on metric and order topologies, Proc. Virginia Polytechnic Institute Conf., 1973.

256. P. R. Meyer and R. Cougar, Topologies of separate continuity in a cross space, Amer. Math. Monthly 75 (1968), 1128 \#5553. 
257. E. Michael, Locally multiplicatively-convex topological algebras, Memoirs Amer. Math. Soc. 11 (1952).

258. _ - Point-finite and locally finite coverings, Canad. J. Math. 7 (1955), 275-279.

259. — A note on closed maps and compact sets, Israel J. Math. 2 (1964), 173-176.

260. ——, $\kappa_{0}$-spaces, J. Math. and Mech. 15 (1966), 983-1002.

261. - Bi-quotient maps and cartesian products of quotient maps, Ann. Inst. Fourier, Grenoble 18 (1968), 287-302.

262. - Local compactness and cartesian products of quotient maps and $k$-spaces, Ann. Inst. Fourier, Grenoble 18 (1968), 281-286.

263. - A note on $k$-spaces and $k_{R}$-spaces, in: Topology Conf., Arizona State Univ., 1967, pp. 247-249.

264. — Bi-sequential spaces and bi-k spaces, address given at the Pittsburgh Intern. Conf. on General Topology, 1970.

265. — On Nagami's $\Sigma$-spaces and some related matters, in: Proc. Washington State Univ. Conf., March 1970, pp. 13-19.

266. - On representing spaces as images of metrizable and related spaces, General Topology and Appl. 1 (1971), 329-343.

267. —, A quintuple quotient quest, General Topology and Appl. 2 (1972), 91-138.

268. - On two theorems of V. V. Filippov, in: General Topology and its Relations to Modern Analysis and Algebra. Proceedings of the Third Prague Topological Symposium, 1971, Academic Press, New York, 1972, pp. 307-308.

269. — Countably bi-quotient maps and A-spaces, Blacksburg Topology Conf., March 1973.

270. - On $k$-spaces, $k_{R}$ spaces and $k(X)$, Pacific J. Math. 47 (1973), 487498.

271. - When are hereditarily quotient maps countably bi-quotient? Preliminary report, Not. Amer. Math. Soc. 20 (1973), A-29 \#73T-G19.

272. A. K. Misra, Chain-net spaces, Not. Amer. Math. Soc. 17 (1970), 839 \#70T-G121.

273. R. C. Moore and S. G. Mrowka, Topologies determined by countable objects, Not. Amer. Math. Soc. 11 (1964), 554 \#614-88.

274. K. Morita, On decomposition spaces of locally compact spaces, Proc. Japan Acad. 32 (1956), 544-548.

275. — - Note on mapping spaces, Proc. Japan Acad. 32 (1956), 671-675.

276. — Some properties of $M$-spaces, Proc. Japan Acad. 43 (1967), 869872.

277. — A survey of the theory of M-spaces, General Topology and Appl. 1 (1971), 49-55.

278. — Results related to closed images of M-spaces. III, Proc. Japan Acad. 48 (1972), 16-20.

279. K. Morita and T. Rishel, Results related to closed images of M-spaces. I and II, Proc. Japan Acad. 47, Suppl. II (1971), 1004-1007 and 1008-1011.

280. S. A. Morris, Local compactness and free products of topological groups, Not. Amer. Math. Soc. 19 (1972), A-721 \#72T-G159.

281. S. A. Morris and E. T. Ordman, Remarks on free products of topological groups. Preliminary report, Not. Amer. Math. Soc. 20 (1973), A-454 \#73T-G81.

282. S. Mrowka, A generalization of a theorem concerning the power of a perfect compact metric space, Bull. Acad. Polon. 6 (1958), 89-93. 
283. - On the potency of compact sets and the first axiom of countability, Bull. Acad. Polon. 6 (1958), 7-9.

284. - Some comments on the class $M$ of cardinals, Bull. Acad. Polon. 17 (1969), 411-414.

285. — Mazur theorem and m-adic spaces, Bull. Acad. Polon. 18 (1970), 299-305.

286. $-\mathcal{R}$-spaces, Acta Math. Acad. Sci. Hungar. 21 (1970), 261-266.

287. S. Mrowka, M. Rajagopalan, and T. Soundararajan, A characterization of compact scattered spaces through chain limits, Preprint.

288. S. B. Nadler, Jr. A note on projections and compactness, Amer. Math. Monthly 73 (1966), 275-276.

289. K. Nagami, Ranges which enable open maps to be compact-covering, General Topology and Appl. 3 (1973), 355-367.

290. J. Nagata, Modern General Topology, American Elsevier, New York, 1968.

291. —-, Mappings and M-spaces, Proc. Japan Acad. 45 (1969), 140-144.

292. — Quotient and bi-quotient spaces of M-spaces, Proc. Japan Acad. 45 (1969), 25-29.

293. S. I. Nedev, Factor images of metric spaces, Soviet Math. Dokl. 9 (1968), 1245-1248.

294. —_, Any linearly ordered symmetrizable space is metrizable, Moscow Univ. Math. Bull. 24 (1969) \#6, 80-81.

295. - Continuous and semicontinuous o-metrics, Soviet Math. Dokl. 11 (1970), 975-978.

296. S. I. Nedev and M. Coban, On metrizability of topological groups, Vestnik Moscov Univ. (1968) \#6, 18-20.

297. — On $\triangle$ - Onetrizable p-spaces, Vestnik Moscov Univ. (1970) \#6, 3-9.

298. - On the theory of O-metrizable spaces. I, II, III, Vestnik Moscov Univ. (1972) \#1, 8-15; \#2, 10-17; \#3, 10-15.

299. N. Noble, $k$-spaces and some generalizations, Ph.D. Thesis, Univ. of Rochester, 1967.

300. - Ascoli theorems and the exponential map, Trans. Amer. Math. Soc. 143 (1969), 393-411.

301. - Countably compact and pseudocompact products, Czech. Math. J. 19 (94) (1969), 390-397.

302. — - A note on z-closed projections, Proc. Amer. Math. Soc. 23 (1969), 73-76.

303. - Products with closed projections, Trans. Amer. Math. Soc. 140 (1969), 381-391.

304. - The continuity of functions on Cartesian products, Trans. Amer. Math. Soc. 149 (1970), 187-198.

305. — $-k$-groups and duality, Trans. Amer. Math. Soc. 151 (1970), 551561 .

306. — Products with closed projections. II, Trans. Amer. Math. Soc. 160 (1971), 169-183.

307. - Products of quotient maps and spaces with weak topologies, Preprint.

308. — - Two examples on preimages of metric spaces, Preprint.

309. — Products of uncountably many $k$-spaces, Proc. Amer. Math. Soc. 31 (1972), 609-612. 
310. J. Novak, On some topological spaces represented by systems of sets, in: Topology and Its Applications. (Proc. of the Adriatic International Symp. held at Herceg-Novi, Yugoslavia, 1968), ed. by D. R. Kurepa, Savez Matematica, 1969, pp. 269-270.

311. A. Okuyama, Note on inverse images under open finite-to-one mappings, Proc. Japan Acad. 43 (1967), 953-956.

312. R. Olson, Bi-quotient maps, countably bi-sequential spaces, and related problems, General Topology and Appl. 4 (1974), 1-28.

313. - On the product of compact Frechet spaces. Preliminary report, Not. Amer. Math. Soc. 20 (1973), A-291 \#73T-G40.

314. P. O'Meara, A metrization theorem, Math. Nachr. 45 (1970), 69-72.

315. J. P. Orevkov, Topological characterization of uniform properties of metric spaces, Soviet Math. Dokl. 4 (1963), 1612-1616.

316. A. J. Ostaszewski, On a question of Arhangel'skii, General Topology and Appl. 3 (1973), 93-95.

317. I. I. Parovicenko, A universal bicompact of weight $\times$, Soviet Math. Dokl. 4 (1963), 592-595.

318. - The branching hypothesis and the correlation between local weight and power to topological spaces, Soviet Math. Dokl. 8 (1967), 589-592.

319. B. A. Pasynkov, Almost metrizable topological groups, Soviet Math. Dokl. 6 (1965), 404-408.

320. T. L. Pearson, Some sufficient conditions for maximal-resolvability, Canad. Math. Bull. 14 (1971), 191-196.

321. V. Ponomarev, Axioms of countability and continuous mapping, Bull. Acad. Polon. 8 (1960), 127-134 (in Russian).

322. - On the cardinality of bicompacta which satisfy the first axiom of countability, Soviet Math. Dokl. 12 (1971), 121-124.

323. W. M. Priestley, Nets and sequences, an example, Amer. Math. Monthly 75 (1968), 1098-1099.

324. - A sequentially closed countable dense subset of $I^{I}$, Proc. Amer. Math. Soc. 24 (1970), 270-271.

325. V. Ptak, On complete topological linear spaces, Czech. Math. J. 78 (1953), 301-364 (Russian with English summary).

326. R. Pupier, Objets convenables, objets de Kelley; application aux espaces topologiques, Rev. Roum. Math. Pures et Appl. 14 (1969), no. 4, 525-532.

327. —, Methodes fonctorielles en topologie generale, Univ. de Lyon, U.E.R. de Math.

329. M. Rajagopalan and T. Soundararajan, Some properties of topological groups, in: General Topology and its Relations to Modern Analysis and Algebra III. Proc. Kanpur Top. Conf. 1968, Prague, 1971.

330. M. J. Reed, Hausdorff-like separation properties and generalizations of the first countability axiom, Preprint.

331. T. W. Rishel, A class of spaces determined by sequences with their cluster points, M. S. Thesis, Univ. of Pittsburgh, 1967.

332. —, A characterization of pseudo-open images of $M$-spaces, Proc. Japan Acad. 45 (1969), 910-912.

333. - - On a subclass of M-spaces, Proc. Japan Acad. 45 (1969), 544-546.

334. — On a subclass of $M$-spaces, II, Not. Amer. Math. Soc. 16 (1969), 767-768 \#667-37.

335. — - A class of spaces determined by sequences with their cluster points, Port. Math. 31 (1972), 187-192. 
336. - Nice spaces; nice maps, in: General Topology and its Relations to Modern Analysis and Algebra. Proceedings of the Third Prague Topological Symposium, 1971, Academic Press, New York, 1972, pp. 375-383.

337. — - A unified technique of proof of some characterization theorems, Preprint.

338. U. Rønnow, Remarks on compact mappings, Math. Scand. 10 (1962), 217220.

339. A. Roux and R. Pupier, Les espaces de Kelley et de de Groot, Applications aux espaces localement compacts, Seminaire de Topologie Generale, Dept. Math., Lyon.

340. P. Roy, The cardinality of first countable spaces, Bull. Amer. Math. Soc. 77 (1971), 1057-1059.

341. L. A. Rubel, Bounded convergence of analytic functions, Bull. Amer. Math. Soc. 77 (1971), 13-24 (p. 19).

342. B. Sapirovskii, On discrete subspaces of topological spaces; weight, tightness and Suslin number, Soviet Math. Dokl. 13 (1972), 215-219.

343. _ - On the density of topological spaces, Soviet Math. Dokl. 13 (1972), 1271-1275.

344. — On separability and metrizability of spaces with Souslin's condition, Soviet Math. Dokl. 13 (1972), 1633-1638.

345. C. T. Scarborough, Closed graphs and closed projections, Proc. Amer. Math. Soc. 20 (1969), 465-470.

346. E. D. Shirley, Pseudo-open maps, Preprint.

347. A. R. Singal, A note on mildly paracompact spaces, Yokohama Math. J. 19 (1971), 1-5.

348. M. K. Singal and M. Deb, On monotone and quasicompact mappings, Israel J. Math. 10 (1971), 218-223.

349. M. K. Singal and A. Mathur, On $E_{1}$-closed and minimal E $E_{1}$-spaces, Glasnik Mat. 6 (26) (1971), 173-178.

350. — - Some weaker forms of countable compactness, Rocky Mountain J. Math. 2 (1972), 569-577.

351. F. Siwiec, KM-spaces and km-covering mappings, Not. Amer. Math. Soc. 18 (1971), 1109-1110 \#71T-G200.

352. - Sequence-covering and countably bi-quotient mappings, General Topology and Appl. 1 (1971), 143-154.

353. - A study of spaces and mappings, including M-spaces, o-spaces, quotient mappings, and A-covering mappings, Ph.D. Thesis, Univ. of Pittsburgh, 1972.

354. — - On defining a space by a weak base, to appear in the Pacific J. Math.

355. - On the theorem of Morita and Hanai, and Stone, Second Pittsburgh International Topology Conf., 1972, Lecture Notes in Math. no. 378, Springer Verlag, New York, 449-454.

356. F. Siwiec and V. J. Mancuso, Relations among certain mappings and conditions for their equivalence, General Topology and Appl. 1 (1971), 33-41.

357. J. C. Smith, Jr., A study of point expandable spaces. Preliminary report, Not. Amer. Math. Soc. 20 (1973), A-175 \# 701-54-22.

358. R. F. Snipes, T-sequential topological spaces, Fund. Math. 77 (1972), 95-98.

359. E. Spanier, Infinite symmetric products, function spaces and duality, Ann. of Math. 69 (1959), 142-198.

360. — Algebraic Topology, McGraw-Hill, New York, 1966.

361. L. A. Steen and J. A. Seebach, Jr., Counterexamples in Topology, Holt, 
Rinehart and Winston, New York, 1970.

362. N. E. Steenrod, A convenient category of topological spaces, Mich. Math. J. 14 (1967), 133-152.

363. R. C. Steinlage, On Ascoli theorems and the product of $k$-spaces, Kyungpook Math. J. 12 (1972), 146-151.

364. R. M. Stephenson, Jr., Pseudocompact spaces, Trans. Aner. Math. Soc. 134 (1968), 437-448.

365. - Product spaces for which the Stone-Weierstrass theorem holds, Proc. Amer. Math. Soc. 21 (1969), 284-288.

366. — - Products of nearly compact spaces, in: Proc. Univ. of Oklahoma Topology Conf., 1972, pp. 310-320.

367. — Product spaces and the Stone-Weierstrass theorem, General Topology and Appl. 3 (1973), 77-79.

368. A. H. Stone, Metrizability of decomposition spaces, Proc. Amer. Math. Soc. 7 (1956), 690-700.

369. G. E. Strecker, E. Wattel, H. Herrlich, and J. de Groot, Strengthening Alexander's subbase theorem, Duke Math. J. 35 (1968), 671-676.

370. P. L. Strong, Quotient and pseudo-open images of separable metric spaces, Proc. Amer. Math. Soc. 33 (1972), 582-586.

371. - Some new classes of mappings between the closed and the pseudoopen mappings, $\mathrm{Ph}$. D. Thesis, Univ. of Illinois, 1972.

372. J. Suzuki, Paracompactness of product spaces, Proc. Japan Acad. 45 (1969), 457-460.

373. F. D. Tall, P-points in $\beta N-N$, normal non-metrizable Moore spaces, and other problems of Hausdorff, Preprint.

374. H. Tamano, A note on the pseudo-compactness of the product of two spaces, Mem. Coll. Sci. Univ. Kyoto, Ser. A 33 (1960), 225-230.

375. Y. Tanaka, On products of quasi-perfect maps, Proc Japan Acad. 46 (1970), 1070-1073.

376. —_ On quasi-k-spaces, Proc Japan Acad. 46 (1970), 1074-1079.

377. — On sequential spaces, Sci. Rep. Tokyo Kyoiku Daigaku Sect. A 11 (1971), 68-72.

378. — On symmetric spaces, Proc. Japan Acad. 49 (1973), 106-111.

379. W. J. Thron, Topological Structures, Holt, Rinehart and Winston, New York, 1966.

380. G. Tironi and R. Isler, On some problems of local approximability in compact spaces, in: General Topology and its Relations to Modern Analysis and Algebra. Proceedings of the Third Prague Topological Symposium, 1971, Academic Press, New York, 1972, pp. 443-446.

381. T'ong, Din' N'E, Preclosed mappings and Taimanov's theorem, Soviet Math. Dokl. 4 (1963), 1335-1338.

382. R. Vaidyanathaswamy, Treatise on Set Topology, Second edition, Chelsea, New York, 1960.

383. K. R. Van Doren, Concerning nonmetrizable images of metrizable spaces under closed, continuous mappings, in: Proc. Houston Topology Conf., 1971, pp. $32-36$.

384. - Closed, continuous images of complete metric spaces, Fund. Math. 80 (1973), 47-50.

385. J. E. Vaughan, Linearly ordered collections and paracompactness, Proc. Amer. Math. Soc. 24 (1970), 186-192.

386. - Spaces of countable and point-countable type, Trans. Amer. Math. Soc. 151 (1970), 341-351. 
387. — Linearly stratifiable spaces, Preprint.

388. G. Vidossich, On compactness in function spaces, Proc. Amer. Math. Soc. 33 (1972), 594-598.

389. A. Volcic, On topologies generated by a subcollection of the collection of closed sets, in: Topology and Its Applications. (Proc. of the Adriatic Intern. Symp. held at Herceg-Novi, Yugoslavia. 1968) ed. by D. R. Kurepa, Savez Matematica, 1969.

390. S. Warner, The topology of compact convergence on continuous function spaces, Duke Math. J. 25 (1958), 265-282.

391. J. H. Webb, Sequential convergence in locally convex spaces, Proc. Camb. Phil. Soc. 64 (1968), 341-364.

392. D. D. Weddington, k-spaces, Ph.D. Thesis, University of Miami, Coral Gables, 1968.

393. — On k-spaces, Proc. Amer. Math. Soc. 22 (1969), 635-638.

394. S. Weingram, Bundles, realizations and $k$-spaces, Ph.D. Thesis, Princeton University, 1961.

395. S. J. Wertheimer, Quasi-compactness and decompositions for arbitrary relations, Pacific J. Math. 37 (1971), 253-263.

396. G. T. Whyburn, Open mappings on locally compact spaces, Amer. Math. Soc. Memoir \# 1 (1950), 1-25.

397. — - Mappings on inverse sets, Duke Math. J. 23 (1956), 237-240.

398. - Directed families of sets and closedness of functions, Proc. Nat. Acad. Sci. U. S. A. 54 (1965), 688-692.

399. — Accessibility spaces, Proc. Amer. Math. Soc. 24 (1970), 181-185.

400. K. Wichterle, On $\mathfrak{B}$-convergence spaces, Czech. Math. J. 18 (93) (1968), 569-588.

401. H. H. Wicke, On the Hausdorff open continuous images of Hausdorff paracompact p-spaces, Proc. Amer. Math. Soc. 22 (1969), 136-140.

402. - Base of countable order theory and some generalizations, in: Proc. Houston Point Set Topology Conf., 1971, pp. 76-95.

403. - Open continuous images of certain kinds of $M$-spaces and completeness of mappings and spaces, General Topology and Appl. 1 (1971), 85-100. 404. A. Wilansky, Between $T_{1}$ and $T_{2}$. Amer. Math. Monthly 74 (1967), 261266.

405. — Life without $T_{2}$. Amer. Math. Monthly 77 (1970), 157-161.

406. _ Topology for Analysis, Ginn, Waltham, 1970.

407. A. Wilansky and H. W. Berkowitz, Metrizable topologies on a Hausdorff space, Amer. Math. Monthly 74 (1967), 1015-1016 \#5396.

408. S. Willard, General Topology, Addison-Wesley, Reading, Mass., 1970.

409. G. K. Williams, Mappings and decompositions, Pacific J. Math. 45 (1973), 387-392.

410. S. W. Williams and S. P. Franklin, Nonregular separable spaces, Amer. Math. Monthly 75 (1968), 208 \#5468.

411. D. E. Wulbert, Subsets of first countable spaces, Proc. Amer. Math. Soc. 19 (1968), 1273-1277.

412. L. G. Zambahidze, On some properties of spaces with bicompact remainders of finite order, Soviet Math. Dokl. 11 (1970), 335-338.

413. P. Zenor, Spaces with subparacompact completions, General Topology and Appl. 3 (1973), 33-38.

414. V. P. Zolotarev, On intersection of topologies, Soviet Math. Dokl. 11 (1970), 1506-1509.

John Jay College of the City University of New York, New York, NY 10019 